\title{
Measuring the Degree of Market Concentration in Thailand Insurance Industry
}

\author{
Sivalap Sukpaiboonwat ${ }^{1}$, Chucheep Piputsitee ${ }^{1} \&$ Arunee Punyasavatsut $^{1}$ \\ ${ }^{1}$ Faculty of Economics, Kasetsart University, Bangkok, Thailand \\ Correspondence: Sivalap Sukpaiboonwat, Faculty of Economics, Kasetsart University, Bangkok, Thailand. \\ E-mail: sivalap@gmail.com
}

Received: October 7, 2013 Accepted: November 29, 2013 Online Published: January 26, 2014

doi:10.5539/ass.v10n4p214 URL: http://dx.doi.org/10.5539/ass.v10n4p214

\begin{abstract}
This paper investigates market structure of life insurance and non-life insurance industry in Thailand. This paper uses the Concentration Ratio and the Herfindahl-Hirschman Index to measure the degree of market concentration. The paper also analyzes various data from all type of insurance premium, balance sheet and income statement to measure the concentration and competition trend.

An analysis of life insurance premium, group market is unconcentrated where as ordinary and industry markets are concentrated. As for the life insurance balance sheet and income statement are concentrated market. An analysis of non-life insurance premium, fire, marine and transportation and automobile markets are unconcentrated. As for the non-life insurance balance sheet and income statement are unconcentrated market. In terms of the same sub-categorized product, personal accident and health insurance have difference concentrate degree. Personal accident in life insurance is near monopoly and highly concentrated market while personal accident in non-life insurance is loose oligopoly and moderate concentrated market. Health insurance in life and non-life insurance are tight oligopoly and concentrated market.

The results conclude that the life insurance industry is more concentrated than the non-life insurance industry. In both segments of the insurance market, as well as in the overall insurance sector, there is a downward trend in market concentration, which indicates the success of the competition promoting process which allows a greater and better choice for customers.
\end{abstract}

Keywords: insurance industry, market structure, concentration measurement

\section{Introduction}

The insurance industry serves individuals and businesses with security products such as life insurance, health insurance, automobile and other liability coverage of non-life insurance. Moreover, insurance industry plays a major role in financial intermediation, thus enhancing a nation's financial and economic development.

Life insurance brings confidence and security for all income groups of society who may be affected by life's uncertainties. At the same time, health insurance has become increasingly important due to the rapidly growing elderly population and the advanced medical technology that enables people to have a longer life span. Developed countries such as the US, Europe, Japan and South Korea as well as developing countries such as Thailand are stepping into the aging society because the elderly population are growing at a faster rate that the child-bearing population. It is estimated that the elderly population in Thailand will increase to 8.0 million (11.9\% of total population) in 2010, to 17.7 million (25.1\% of total population) in 2030 (Office of the National Economic and Social Development Board, 2010). Thus, governments in most countries are making effort in encouraging the population to seek ways to increasing their savings through government bonds, real estate schemes and retirement plans and to boost confidence and security in the form of life and health insurance. With a reliable saving plan, our stable income can be fortified through a life insurance system that provides protection and secures our savings. If the individual learns to take advantage of a savings plan, the saving plan will help increase the country's savings and further lead to the nation's economic development and expansion.

Likewise, non-life insurance is beneficial to business and investment sectors of society who are inevitably faced with daily risks from unforeseen circumstances or natural disasters such as floods, hurricanes, tsunamis, fire, explosions, accidents arising from travel and carelessness. All these risks and financial damage can be alleviated 
by reliable non-life insurance system.

As alluded to above, there were empirical studies that reveal the insurance industry in the term of insurance market structure. Jedlicka \& Jumah (2006) analyze the Austrian insurance industry using insurance premium. The study finds a positive relationship between market share and profitability. The higher market concentration leads to higher market power and higher returns. Dumm \& Macpherson (2006) examine insurance premium in Florida. The study finds that Florida insurance industry is highly concentrated that two companies have 50 percent of premiums and six companies have 99 percent of premiums in 2004. Glambotskaya (2009) studies the structure of the Belarusian insurance market by insurance premiums. The study finds that Belarusian insurance business concentration is very high. Herfindal-Hirshman index for the insurance sector in 2007, calculated on the written premiums, was 3540. The biggest share of the insurance market belongs to the state-owned insurers. Austin \& Hungerford (2009) study the market structure of the health insurance industry in United State. The study finds that health insurance markets are highly concentrated. The concentrated markets lead to high premium and limit access to health insurance. All of the studies show insurance market having a high market share and high market power.

Past studies concentrated only on insurance premium. No study investigates in the part of balance sheet and income statement, in spite of the fact that these data are keys to the financial position. Thus, this paper use balance sheet and income statement to measure market structure. This study will determine all type of premium both of life and non-life insurance and learn more about balance sheet which include assets, liabilities and shareholder's equity and income statement which include revenue, expenditures and net profit for the most comprehensive and complete insurance market study. It is helpful to have an understanding of the current structure of the insurance industry and consider their future paths. This study is organized as follows: section 2 measures the degree of concentration in Thailand insurance industry; section 3 shows the results of market concentration in Thailand insurance industry; then section 4 presents conclusions of the study.

\section{Method}

A market is any arrangement in which buyers and sellers exchange goods or services at agreed-upon price and quantity. Market structure is implying the number and relative size of the producers in an industry. An industry's market structure is a reflection the similarity of products produced within the industry and the relative ease for new producers to enter the market. Market structure also determines the producers' competitive environment. Economists commonly categorize market structures into four basic types: perfect competition, monopoly, monopolistic competition, and oligopoly (Johnson \& Stone, 1998).

The imperfect market such as monopolistic competition, oligopoly, and monopoly, which there are few sellers and differentiated products, results in some market power. On the opposite, perfect competition, a market structure characterized by a large number of small sellers supplying a homogeneous product incurs no market power. To consider size of firm and significant percentage of the market supply, there are two elements of market structure. They are concentration ratios and Herfindahl-Hirschman index.

The concentration ratio is the percentage of market share owned by the largest $\mathrm{n}$ firms in an industry. The largest firm concentration ratio is $\mathrm{CR}_{1}$. The most common concentration ratio is the four-firm concentration ratio $\left(\mathrm{CR}_{4}\right)$.

Let $S_{i}$ denotes the output of each firms in an industry, $(i=1,2,3 \ldots n)$, and $S_{T}$ denotes the total output of all firms in the industry. Let firm i's share of the total market output is $\mathrm{W}_{\mathrm{i}}=\mathrm{S}_{\mathrm{i}} / \mathrm{S}_{\mathrm{T}}$. Then the $\mathrm{CR}_{4}$ is the fraction of total industry output produced by the four largest firms in the industry.

$$
C R_{4}=\frac{s_{1}+s_{2}+s_{3}+s_{4}}{s_{T}}, C R_{4}=\sum_{i=1}^{4} w_{i}
$$

Another measurement is Herfindahl-Hirschman index (HHI). The HHI is the sum of the squared market shares of firms in the industry, multiplied by 10000 to eliminate the decimals. Then the Herfindahl-Hirschman index is

$$
\mathrm{HHI}=10,000\left[\left(\frac{s_{1}}{s_{T}}\right)^{2}+\left(\frac{s_{2}}{s_{T}}\right)^{2}+\ldots+\left(\frac{s_{n}}{s_{T}}\right)^{2}\right], \mathrm{HHI}=10,000 \sum_{i=1}^{n}{\mathcal{W}_{i}^{2}}^{2}
$$

The HHI measures the size of firms in relation to the industry, but also as an indication of the amount of competition. If HHI decrease it is interpreted as a loss of market power and at the same time an increase in competition. 
Table 1. Classifying industries with the $\mathrm{CR}_{1}, \mathrm{CR}_{4}$, and $\mathrm{HHI}$

\begin{tabular}{llll}
\hline $\mathrm{CR}_{4}$ & $\mathrm{HHI}$ & Interpretation of Market Structure & \\
\hline $\mathrm{CR}_{4}=0$ & $\mathrm{HHI}=0$ & $\begin{array}{l}\text { Perfect Competition } \\
\text { Effective Competition or } \quad \text { Monopolistic }\end{array}$ & Competition, \\
$0<\mathrm{CR}_{4}<40$ & $\mathrm{HHI}<1000$ & $\begin{array}{l}\text { Unconcentrated } \\
\text { Loose Oligopoly or Monopolistic Competition, Moderately } \\
\text { concentrated }\end{array}$ \\
$40 \leq \mathrm{CR}_{4}<60$ & $1000 \leq \mathrm{HHI}<1800$ & Tight Oligopoly, Concentrated \\
$60 \leq \mathrm{CR}_{4}<90$ & $1800 \leq \mathrm{HHI}<2500$ & Effective Monopoly (near monopoly), Highly concentrated \\
$90 \leq \mathrm{CR}_{4}$ & $2500 \leq \mathrm{HHI}<9999$ & Monopoly \\
$\mathrm{CR}_{1}=100$ & $\mathrm{HHI}=10000$ & &
\end{tabular}

Source: Lipczynski et al. (2005, p. 215)

Table 2. Comparison of market structures

\begin{tabular}{|c|c|c|c|c|}
\hline $\begin{array}{l}\text { Type or Market } \\
\text { Structure }\end{array}$ & $\begin{array}{l}\text { Power to set supply } \\
\text { and price }\end{array}$ & $\begin{array}{l}\text { Number } \\
\text { sellers }\end{array}$ & $\begin{array}{l}\text { Level of product } \\
\text { differentiation }\end{array}$ & $\begin{array}{l}\text { Number of barriers } \\
\text { to entry }\end{array}$ \\
\hline Monopoly & High & One & Unique & Many \\
\hline Oligopoly & Some & Few & $\begin{array}{l}\text { Either differentiated or } \\
\text { homogeneous }\end{array}$ & Many \\
\hline $\begin{array}{l}\text { Monopolistic } \\
\text { Competition }\end{array}$ & Very little & Many & Differentiated & Few \\
\hline Perfect Competition & None & Very many & Homogeneous & None \\
\hline
\end{tabular}

Source: Johnson and Stone (1998, p. 64)

\section{Results}

The prevailing method of analyzing market structure is the measurement of concentration. This section focuses on life and non-life insurance industry in term of the $\mathrm{CR}_{1}$ ratio, $\mathrm{CR}_{4}$ ratio and Herfindahl-Hirschman index.

\subsection{Life Insurance Industry}

This part presents market concentration on life insurance premium, life insurance balance sheet and life insurance income statement

3.1.1 Market Concentration on Life Insurance Premium

Table 3. Market concentration on life insurance premium

\begin{tabular}{|c|c|c|c|c|c|c|c|c|c|c|}
\hline \multicolumn{2}{|l|}{ Year } & \multicolumn{2}{|l|}{1997} & \multicolumn{2}{|l|}{1998} & \multicolumn{2}{|l|}{2010} & 2011 & \multicolumn{2}{|c|}{$\begin{array}{l}\text { \% increase } \\
\text { (decrease) }\end{array}$} \\
\hline \multicolumn{11}{|c|}{ 1. Total Premium } \\
\hline $\mathrm{CR}_{1}$ & 46.83 & $\begin{array}{l}\text { moderate } \\
\text { market share }\end{array}$ & 47.50 & $\begin{array}{l}\text { moderate } \\
\text { market share }\end{array}$ & 30.93 & $\begin{array}{l}\text { moderate } \\
\text { market share }\end{array}$ & 28.79 & $\begin{array}{l}\text { low } \\
\text { share }\end{array}$ & market & $(6.91)$ \\
\hline $\mathrm{CR}_{4}$ & 85.33 & tight oligopoly & 85.48 & tight oligopoly & 62.24 & tight oligopoly & 62.08 & tight ol & sopoly & $(0.26)$ \\
\hline HHI & 2900 & $\begin{array}{l}\text { highly } \\
\text { concentrated }\end{array}$ & 2941 & $\begin{array}{l}\text { highly } \\
\text { concentrated }\end{array}$ & 1470 & $\begin{array}{l}\text { moderate } \\
\text { concentrated }\end{array}$ & 1402 & $\begin{array}{l}\text { modera } \\
\text { concen }\end{array}$ & ated & $(4.63)$ \\
\hline \multicolumn{11}{|c|}{ 2. Ordinary premium } \\
\hline $\mathrm{CR}_{1}$ & 54.3 & $\begin{array}{l}\text { high market } \\
\text { share }\end{array}$ & 55.13 & $\begin{array}{l}\text { high market } \\
\text { share }\end{array}$ & 33.54 & $\begin{array}{l}\text { moderate } \\
\text { market share }\end{array}$ & 30.29 & $\begin{array}{l}\text { modera } \\
\text { market }\end{array}$ & hare & $(9.68)$ \\
\hline $\mathrm{CR}_{4}$ & 90.13 & near monopoly & 90.56 & near monopoly & 67.11 & tight oligopoly & 65.45 & tight ol & ropoly & $(2.47)$ \\
\hline HHI & 3597 & $\begin{array}{l}\text { highly } \\
\text { concentrated }\end{array}$ & 3664 & $\begin{array}{l}\text { highly } \\
\text { concentrated }\end{array}$ & 1682 & $\begin{array}{l}\text { moderate } \\
\text { concentrated }\end{array}$ & 1522 & $\begin{array}{l}\text { modera } \\
\text { concen }\end{array}$ & ated & $(9.51)$ \\
\hline
\end{tabular}




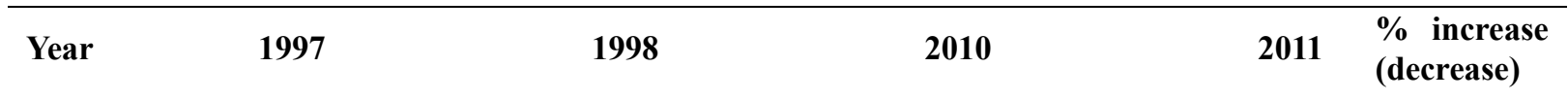

\section{Industry Premium}

\begin{tabular}{|c|c|c|c|c|c|c|c|c|c|}
\hline $\mathrm{CR}_{1}$ & 65.08 & $\begin{array}{l}\text { high } \\
\text { share }\end{array}$ & 66.61 & $\begin{array}{l}\text { high } \\
\text { share }\end{array}$ & 79.27 & $\begin{array}{l}\text { high } \\
\text { share }\end{array}$ & 78.04 & $\begin{array}{l}\text { high } \\
\text { share }\end{array}$ & $(1.55)$ \\
\hline $\mathrm{CR}_{4}$ & 98.63 & near monopoly & 98.57 & near monopoly & 99.73 & near monopoly & 99.75 & near monopoly & 0.02 \\
\hline HHI & 5046 & $\begin{array}{l}\text { highly } \\
\text { concentrated }\end{array}$ & 5196 & $\begin{array}{l}\text { highly } \\
\text { concentrated }\end{array}$ & 6516 & $\begin{array}{l}\text { highly } \\
\text { concentrated }\end{array}$ & 6348 & $\begin{array}{l}\text { highly } \\
\text { concentrated }\end{array}$ & $(2.58)$ \\
\hline
\end{tabular}

\section{Group Premium}

$\begin{array}{lcll}\mathrm{CR}_{1} & 33.83 & \begin{array}{l}\text { moderate } \\ \text { market share }\end{array} \\ \mathrm{CR}_{4} & 70.14 & \text { tight oligopoly } \\ & & 69.4 \\ \mathrm{HHI} & 1738 & \begin{array}{l}\text { moderate } \\ \text { concentrated }\end{array}\end{array}$

\section{Personal Accident Premium}

$\mathrm{CR}_{1}$

$\mathrm{CR}_{4}$

n. a.

n. a. moderate market share

tight oligopoly

moderate

concentrated
15.27

49.76

903

low market

share

loose oligopoly

unconcentrated

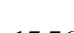

17.76

low market

share

52.17 loose oligopoly

958

unconcentrated

HHI

\section{Health Insurance Premium}

\begin{tabular}{|c|c|c|c|c|c|c|c|c|c|}
\hline $\mathrm{CR}_{1}$ & 52.65 & $\begin{array}{l}\text { high market } \\
\text { share }\end{array}$ & 52.52 & market & 49.34 & $\begin{array}{l}\text { moderate } \\
\text { market share }\end{array}$ & 48.23 & $\begin{array}{l}\text { moderate } \\
\text { market share }\end{array}$ & $(2.25)$ \\
\hline $\mathrm{CR}_{4}$ & 92.18 & Near monopoly & 91.70 & Near monopoly & 83.79 & Tight oligopoly & 83.13 & Tight oligopoly & $(0.79)$ \\
\hline HHI & 3583 & $\begin{array}{l}\text { Highly } \\
\text { concentrated }\end{array}$ & 3507 & $\begin{array}{l}\text { Highly } \\
\text { concentrated }\end{array}$ & 2906 & $\begin{array}{l}\text { Highly } \\
\text { concentrated }\end{array}$ & 2804 & $\begin{array}{l}\text { Highly } \\
\text { concentrate }\end{array}$ & $(3.51)$ \\
\hline
\end{tabular}

Source: Calculated by author, using data from Office of Insurance Commission

\begin{tabular}{|c|c|c|c|c|}
\hline 74.55 & market & 74.45 & $\begin{array}{l}\text { high } \\
\text { share }\end{array}$ & $(0.14)$ \\
\hline 90.13 & Near monopoly & 91.76 & Near monopoly & 1.80 \\
\hline 5664 & $\begin{array}{l}\text { highly } \\
\text { concentrated }\end{array}$ & 5662 & $\begin{array}{l}\text { highly } \\
\text { concentrated }\end{array}$ & $(0.04)$ \\
\hline
\end{tabular}
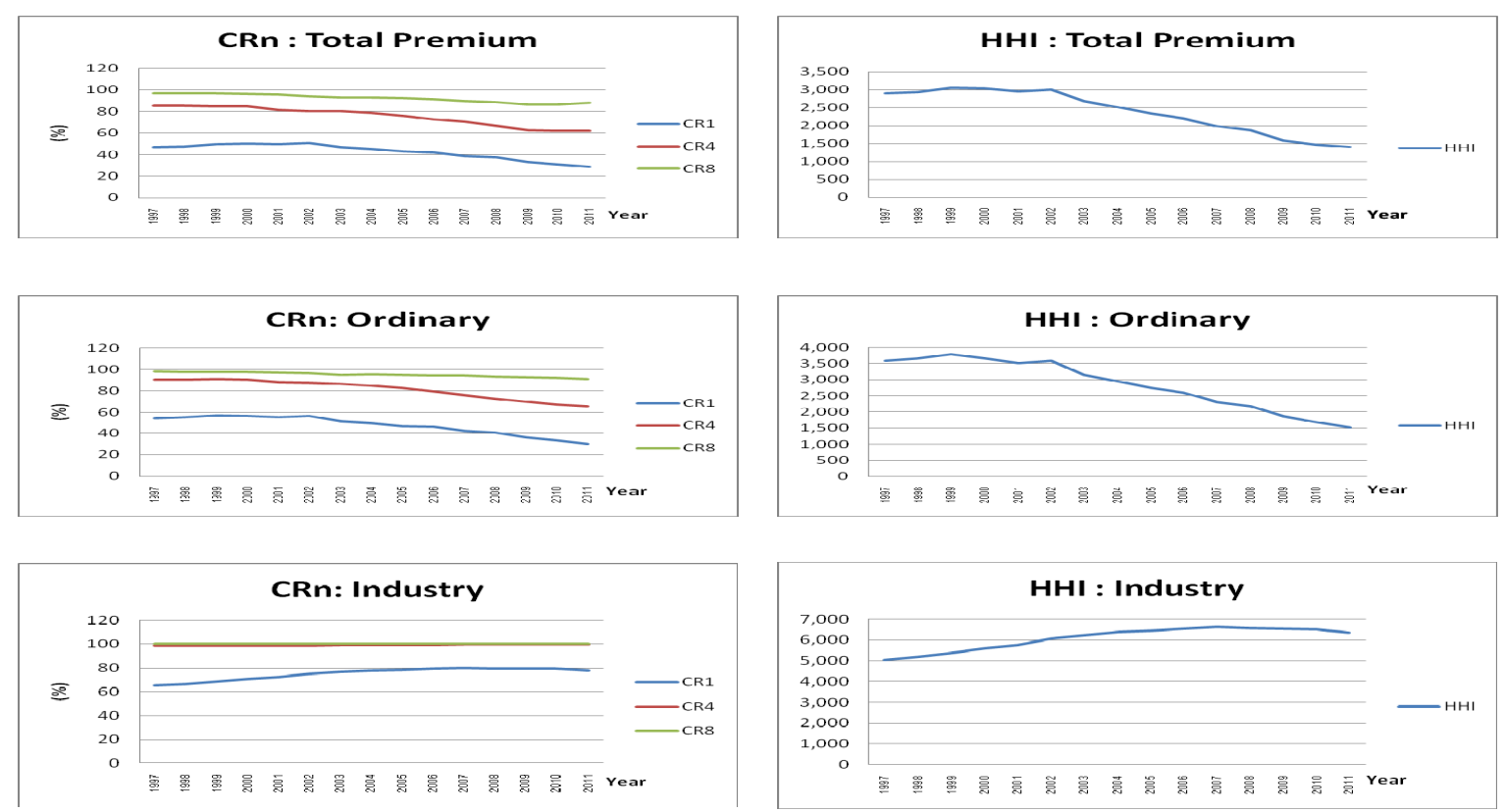

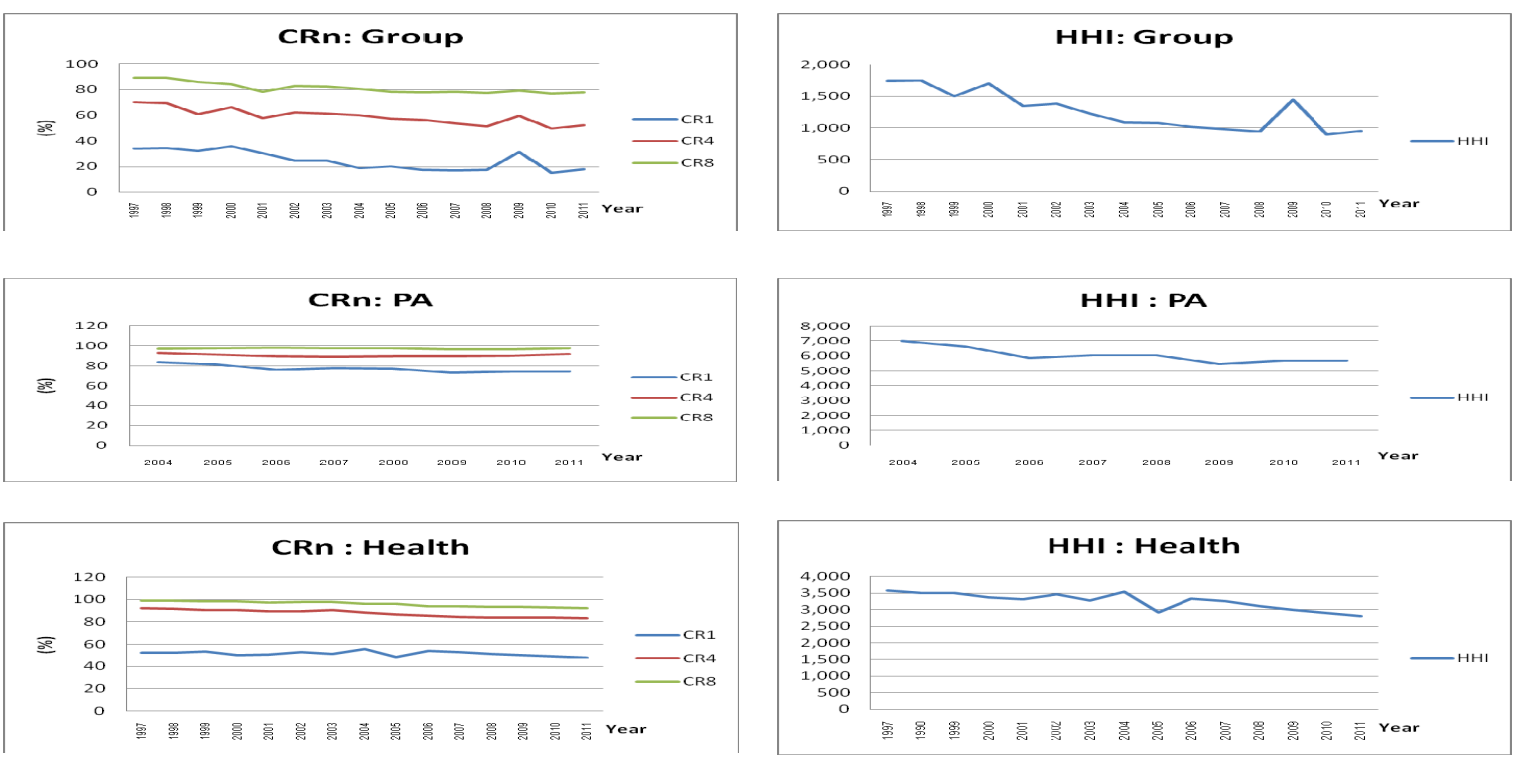

Figure 1. Market concentrations on direct life insurance premium

Source: Calculated by author, using data from Office of Insurance Commission

As shown in Table 3 and Figure 1, the life insurance industry in term of total premium indicates tight oligopoly and concentrated market with decreasing trend. The $\mathrm{CR}_{4}$ and $\mathrm{HHI}$ of total premium are $62.08 \%$ and 1402 , respectively, in 2011. Life insurance industry in Thailand has four sub-categorized products, including ordinary, industry, group, personal accident and health insurance product. The highest competition is group product and highest concentration is industry product. The $\mathrm{CR}_{4}$ of group premium is $17.76 \%$ and the $\mathrm{CR}_{4}$ of industry premium is $78.04 \%$ in 2011 . The HHI of group premium is 958 and the HHI of industry premium is 6348 in 2011.

3.1.2 Market Concentration on Life Insurance Balance Sheet

Table 4. Market concentration on life insurance balance sheet

\begin{tabular}{|c|c|c|c|c|c|}
\hline Year & 2006 & 2007 & 2010 & 2011 & $\begin{array}{l}\text { \% increase } \\
\text { (decrease) }\end{array}$ \\
\hline
\end{tabular}

\section{Investment Assets}

\begin{tabular}{|c|c|c|c|c|c|c|c|c|}
\hline $\mathrm{CR}_{1}$ & 48.20 & $\begin{array}{l}\text { moderate } \\
\text { market share }\end{array}$ & 47.18 & $\begin{array}{l}\text { moderate } \\
\text { market share }\end{array}$ & 42.09 & $\begin{array}{l}\text { moderate } \\
\text { market share }\end{array}$ & 39.80 & $\begin{array}{l}\text { moderate } \\
\text { market share }\end{array}$ \\
\hline $\mathrm{CR}_{4}$ & 80.16 & tight oligopoly & 78.34 & tight oligopoly & 71.00 & tight oligopoly & 68.79 & $\begin{array}{l}\text { tight } \\
\text { oligopoly }\end{array}$ \\
\hline HHI & 2771 & $\begin{array}{l}\text { highly } \\
\text { concentrated }\end{array}$ & 2668 & $\begin{array}{l}\text { highly } \\
\text { concentrated }\end{array}$ & 2195 & concentrated & 2023 & concentrated \\
\hline
\end{tabular}

\section{Building \& Operating Assets}

\begin{tabular}{|c|c|c|c|c|c|c|c|c|c|}
\hline $\mathrm{CR}_{1}$ & 31.12 & $\begin{array}{l}\text { moderate } \\
\text { market share }\end{array}$ & 31.02 & $\begin{array}{l}\text { moderate } \\
\text { market share }\end{array}$ & 33.39 & $\begin{array}{l}\text { moderate } \\
\text { market share }\end{array}$ & 29.98 & $\begin{array}{l}\text { low market } \\
\text { share }\end{array}$ & (10.20) \\
\hline $\mathrm{CR}_{4}$ & 85.86 & tight oligopoly & 82.28 & tight oligopoly & 84.82 & tight oligopoly & 85.74 & $\begin{array}{l}\text { tight } \\
\text { oligopoly }\end{array}$ & 1.08 \\
\hline HHI & 2233 & concentrated & 2087 & concentrated & 2219 & concentrated & 2278 & concentrated & 2.65 \\
\hline \multicolumn{10}{|c|}{ 3. Others Assets } \\
\hline $\mathrm{CR}_{1}$ & 34.34 & $\begin{array}{l}\text { moderate } \\
\text { market share }\end{array}$ & 33.45 & $\begin{array}{l}\text { moderate } \\
\text { market share }\end{array}$ & 23.20 & market & 25.54 & $\begin{array}{l}\text { low market } \\
\text { share }\end{array}$ & 10.08 \\
\hline
\end{tabular}




\begin{tabular}{|c|c|c|c|c|c|c|c|c|c|}
\hline Year & & 2006 & & 2007 & & 2010 & & 2011 & $\begin{array}{l}\text { \% increase } \\
\text { (decrease) }\end{array}$ \\
\hline $\mathrm{CR}_{4}$ & 66.97 & tight oligopoly & 65.33 & tight oligopoly & 60.48 & tight oligopoly & 60.92 & $\begin{array}{l}\text { tight } \\
\text { oligopoly }\end{array}$ & 0.72 \\
\hline HHI & 1690 & $\begin{array}{l}\text { moderate } \\
\text { concentrated }\end{array}$ & 1626 & $\begin{array}{l}\text { moderate } \\
\text { concentrated }\end{array}$ & 1207 & $\begin{array}{l}\text { moderate } \\
\text { concentrated }\end{array}$ & 1294 & $\begin{array}{l}\text { moderate } \\
\text { concentrate }\end{array}$ & 7.22 \\
\hline
\end{tabular}

\section{Total Asset}

\begin{tabular}{|c|c|c|c|c|c|c|c|c|}
\hline $\mathrm{CR}_{1}$ & 47.10 & $\begin{array}{l}\text { moderate } \\
\text { market share }\end{array}$ & 46.13 & $\begin{array}{l}\text { moderate } \\
\text { market share }\end{array}$ & 40.96 & $\begin{array}{l}\text { moderate } \\
\text { market share }\end{array}$ & 38.82 & $\begin{array}{l}\text { moderate } \\
\text { market share }\end{array}$ \\
\hline $\mathrm{CR}_{4}$ & 79.50 & tight oligopoly & 77.73 & tight oligopoly & 70.39 & tight oligopoly & 68.41 & $\begin{array}{l}\text { tight } \\
\text { oligopoly }\end{array}$ \\
\hline HHI & 2677 & $\begin{array}{l}\text { highly } \\
\text { concentrated }\end{array}$ & 2580 & $\begin{array}{l}\text { highly } \\
\text { concentrated }\end{array}$ & 2115 & concentrated & 1962 & concentrated \\
\hline
\end{tabular}

\section{Policy Liabilities}

\begin{tabular}{|c|c|c|c|c|c|c|c|c|}
\hline $\mathrm{CR}_{1}$ & 42.70 & $\begin{array}{l}\text { moderate } \\
\text { market share }\end{array}$ & 41.42 & $\begin{array}{l}\text { moderate } \\
\text { market share }\end{array}$ & 37.93 & $\begin{array}{l}\text { moderate } \\
\text { market share }\end{array}$ & 34.57 & $\begin{array}{l}\text { moderate } \\
\text { market share }\end{array}$ \\
\hline $\mathrm{CR}_{4}$ & 78.86 & tight oligopoly & 76.75 & tight oligopoly & 69.10 & tight oligopoly & 66.15 & $\begin{array}{l}\text { tight } \\
\text { oligopoly }\end{array}$ \\
\hline HHI & 2380 & concentrated & 2265 & concentrated & 1929 & concentrated & 1718 & $\begin{array}{l}\text { moderate } \\
\text { concentrated }\end{array}$ \\
\hline
\end{tabular}

\section{Other Liabilities}

\begin{tabular}{|c|c|c|c|c|c|c|c|c|c|}
\hline $\mathrm{CR}_{1}$ & 58.90 & $\begin{array}{l}\text { high market } \\
\text { share }\end{array}$ & 59.17 & $\begin{array}{l}\text { high market } \\
\text { share }\end{array}$ & 45.88 & $\begin{array}{l}\text { moderate } \\
\text { market share }\end{array}$ & 40.03 & $\begin{array}{l}\text { moderate } \\
\text { market share }\end{array}$ & $(12.76)$ \\
\hline $\mathrm{CR}_{4}$ & 82.69 & tight oligopoly & 79.99 & tight oligopoly & 66.89 & tight oligopoly & 64.23 & $\begin{array}{l}\text { tight } \\
\text { oligopoly }\end{array}$ & $(3.98)$ \\
\hline HHI & 3706 & $\begin{array}{l}\text { highly } \\
\text { concentrated }\end{array}$ & 3707 & $\begin{array}{l}\text { highly } \\
\text { concentrated }\end{array}$ & 2370 & concentrated & 1953 & concentrated & (17.59) \\
\hline \multicolumn{10}{|c|}{ 7. Capital Funds } \\
\hline $\mathrm{CR}_{1}$ & 68.69 & $\begin{array}{l}\text { high market } \\
\text { share }\end{array}$ & 68.20 & market & 57.25 & $\begin{array}{l}\text { high market } \\
\text { share }\end{array}$ & 53.03 & $\begin{array}{l}\text { high market } \\
\text { share }\end{array}$ & (22.79) \\
\hline $\mathrm{CR}_{4}$ & 87.12 & tight oligopoly & 88.49 & tight oligopoly & 81.28 & tight oligopoly & 79.22 & $\begin{array}{l}\text { tight } \\
\text { oligopoly }\end{array}$ & $(9.07)$ \\
\hline HHI & 4868 & $\begin{array}{l}\text { highly } \\
\text { concentrated }\end{array}$ & 4817 & $\begin{array}{l}\text { highly } \\
\text { concentrated }\end{array}$ & 3524 & $\begin{array}{l}\text { highly } \\
\text { concentrated }\end{array}$ & 3109 & $\begin{array}{l}\text { highly } \\
\text { concentrated }\end{array}$ & (36.13) \\
\hline
\end{tabular}

Source: Calculated by author, using data from Office of Insurance Commission
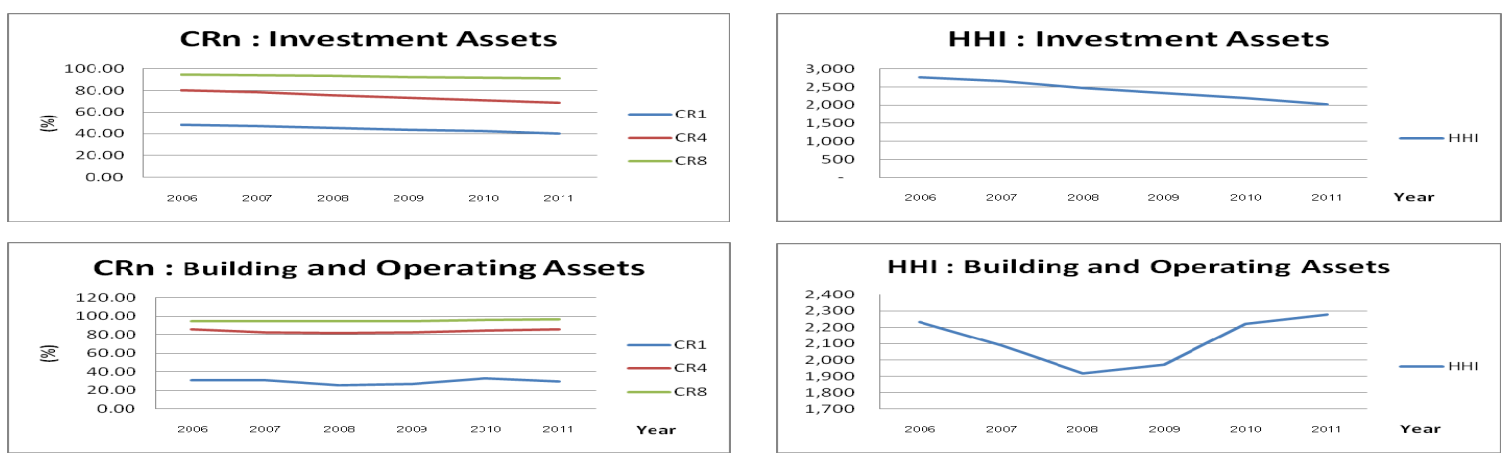

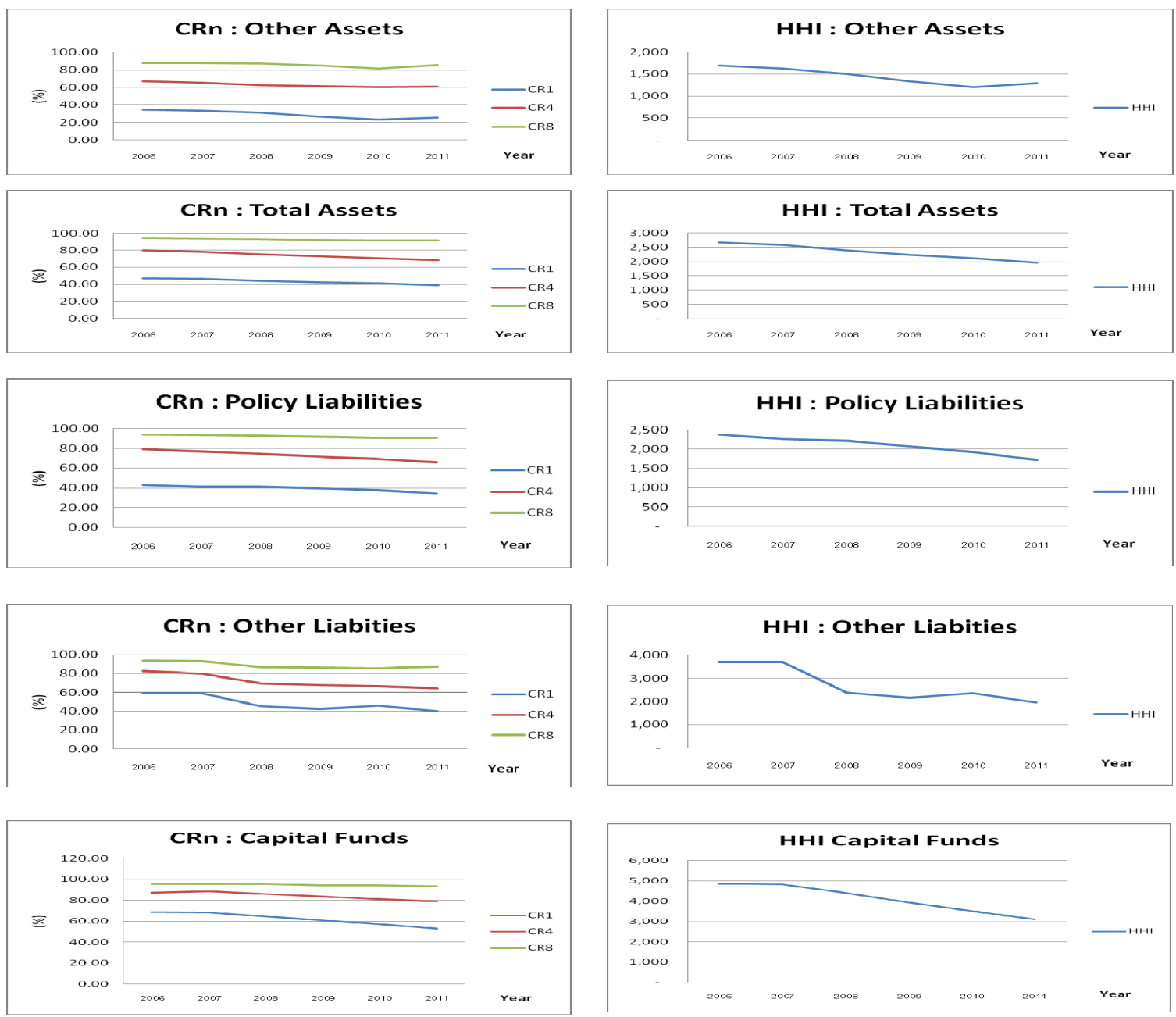

Figure 2. Market concentration on life insurance balance sheet

Source: Calculated by author, using data from Office of Insurance Commission

As shown in Table 4 and Figure 2, the life insurance balance sheet is tight oligopoly and concentrated. The $\mathrm{CR}_{4}$ and $\mathrm{HHI}$ of investment asset are $68.79 \%$ and 2023, respectively, in 2011 . The $\mathrm{CR}_{4}$ and $\mathrm{HHI}$ of total asset are $68.41 \%$ and 1962, respectively, in 2011. The $\mathrm{CR}_{4}$ and HHI of policy liabilities are $66.15 \%$ and 1718 , respectively, in 2011. The $\mathrm{CR}_{4}$ and $\mathrm{HHI}$ of capital funds are 79.22\% and 3109, respectively, in 2011.

3.1.3 Market Concentration on Life Insurance Income Statement

Table 5. Market concentration on life insurance income statement

\begin{tabular}{|c|c|c|c|c|c|c|c|c|c|}
\hline \multicolumn{2}{|l|}{ Year } & \multicolumn{2}{|l|}{2006} & \multicolumn{2}{|l|}{2007} & \multicolumn{2}{|l|}{2010} & \multicolumn{2}{|c|}{$\begin{array}{l}\text { \% increase } \\
\text { (decrease) }\end{array}$} \\
\hline \multicolumn{10}{|c|}{ 1. Net Written Premiums } \\
\hline CR1 & 41.87 & $\begin{array}{l}\text { moderate } \\
\text { market share }\end{array}$ & 38.74 & $\begin{array}{l}\text { moderate } \\
\text { market share }\end{array}$ & 30.95 & $\begin{array}{l}\text { moderate } \\
\text { market share }\end{array}$ & 28.82 & $\begin{array}{l}\text { low market } \\
\text { share }\end{array}$ & $(6.87)$ \\
\hline CR4 & 72.70 & tight oligopoly & 70.45 & tight oligopoly & 62.48 & tight oligopoly & 62.21 & tight oligopoly & $(0.44)$ \\
\hline HHI & 2211 & concentrated & 1992 & concentrated & 1476 & $\begin{array}{l}\text { moderate } \\
\text { concentrated }\end{array}$ & 1407 & $\begin{array}{l}\text { moderate } \\
\text { concentrated }\end{array}$ & $(4.72)$ \\
\hline \multicolumn{10}{|c|}{ 2. Net Investment Income \& Other Income } \\
\hline CR1 & 52.26 & $\begin{array}{l}\text { high } \\
\text { share }\end{array}$ & 49.36 & $\begin{array}{l}\text { moderate } \\
\text { market share }\end{array}$ & 43.82 & $\begin{array}{l}\text { moderate } \\
\text { market share }\end{array}$ & 39.70 & $\begin{array}{l}\text { moderate } \\
\text { market share }\end{array}$ & $(9.42)$ \\
\hline
\end{tabular}




\begin{tabular}{|c|c|c|c|c|c|c|c|c|c|}
\hline Year & & 2006 & & 2007 & & 2010 & & 2011 & $\begin{array}{l}\text { \% increase } \\
\text { (decrease) }\end{array}$ \\
\hline CR4 & 81.75 & tight oligopoly & 80.06 & tight oligopoly & 72.36 & tight oligopoly & 69.18 & tight oligopoly & $(4.40)$ \\
\hline HHI & 3103 & $\begin{array}{l}\text { highly } \\
\text { concentrated }\end{array}$ & 2849 & $\begin{array}{l}\text { highly } \\
\text { concentrated }\end{array}$ & 2338 & concentrated & 2036 & concentrated & $(12.91)$ \\
\hline \multicolumn{10}{|c|}{ 3.Total Income } \\
\hline CR1 & 43.60 & $\begin{array}{l}\text { moderate } \\
\text { market share }\end{array}$ & 40.46 & $\begin{array}{l}\text { moderate } \\
\text { market share }\end{array}$ & 32.93 & $\begin{array}{l}\text { moderate } \\
\text { market share }\end{array}$ & 30.52 & $\begin{array}{l}\text { moderate } \\
\text { market share }\end{array}$ & $(7.31)$ \\
\hline CR4 & 73.73 & tight oligopoly & 71.66 & tight oligopoly & 63.79 & tight oligopoly & 63.27 & tight oligopoly & $(0.80)$ \\
\hline HHI & 2340 & concentrated & 2111 & concentrated & 1580 & $\begin{array}{l}\text { moderate } \\
\text { concentrated }\end{array}$ & 1483 & $\begin{array}{l}\text { moderate } \\
\text { concentrated }\end{array}$ & (6.18) \\
\hline
\end{tabular}

\section{Life Policy Reserve}

\begin{tabular}{|c|c|c|c|c|}
\hline CR1 & 33.96 & $\begin{array}{l}\text { moderate } \\
\text { market share }\end{array}$ & 32.98 & $\begin{array}{l}\text { moderate } \\
\text { market share }\end{array}$ \\
\hline CR4 & 68.70 & tight oligopoly & 66.86 & tight oligopoly \\
\hline HHI & 1755 & $\begin{array}{l}\text { moderate } \\
\text { concentrated }\end{array}$ & 1649 & $\begin{array}{l}\text { moderate } \\
\text { concentrated }\end{array}$ \\
\hline
\end{tabular}

\section{Benefit Payments During the Year}

$\begin{array}{llll}\text { CR1 } & 48.87 & \begin{array}{l}\text { moderate } \\ \text { market share }\end{array} & 43.7 \\ \text { CR4 } & 80.55 & \text { tight oligopoly } & 79.07 \\ \text { HHI } & 2911 & \begin{array}{l}\text { highly } \\ \text { concentrated }\end{array} & 2527 \\ & & \end{array}$

\begin{tabular}{|c|c|c|c|c|c|}
\hline 43.77 & $\begin{array}{l}\text { moderate } \\
\text { market share }\end{array}$ & 39.76 & $\begin{array}{l}\text { moderate } \\
\text { market share }\end{array}$ & 38.00 & $\begin{array}{l}\text { moderate } \\
\text { market share }\end{array}$ \\
\hline 79.07 & tight oligopoly & 72.88 & tight oligopoly & 71.75 & tight oligopoly \\
\hline 2527 & $\begin{array}{l}\text { highly } \\
\text { concentrated }\end{array}$ & 2137 & concentrated & 2015 & concentrated \\
\hline
\end{tabular}

\section{Dividends}

\begin{tabular}{|c|c|c|c|c|c|c|c|c|c|}
\hline CR1 & 64.90 & $\begin{array}{l}\text { high } \\
\text { share }\end{array}$ & 55.47 & market & 33.40 & $\begin{array}{l}\text { moderate } \\
\text { market share }\end{array}$ & 31.66 & $\begin{array}{l}\text { moderate } \\
\text { market share }\end{array}$ & $(5.20)$ \\
\hline CR4 & 96.75 & near monopoly & 95.43 & near monopoly & 93.81 & near monopoly & 97.43 & near monopoly & 3.87 \\
\hline HHI & 4717 & $\begin{array}{l}\text { highly } \\
\text { concentrated }\end{array}$ & 3807 & $\begin{array}{l}\text { highly } \\
\text { concentrated }\end{array}$ & 2381 & concentrated & 2492 & concentrated & 4.66 \\
\hline \multicolumn{10}{|c|}{ 7. Salary and Brokerages } \\
\hline CR1 & 44.88 & $\begin{array}{l}\text { moderate } \\
\text { market share }\end{array}$ & 45.51 & $\begin{array}{l}\text { moderate } \\
\text { market share }\end{array}$ & 24.16 & $\begin{array}{l}\text { low market } \\
\text { share }\end{array}$ & 37.02 & $\begin{array}{l}\text { moderate market } \\
\text { share }\end{array}$ & 53.21 \\
\hline CR4 & 72.79 & tight oligopoly & 71.45 & tight oligopoly & 60.71 & tight oligopoly & 69.56 & tight oligopoly & 14.58 \\
\hline HHI & 2413 & concentrated & 2425 & concentrated & 1230 & $\begin{array}{l}\text { moderate } \\
\text { concentrated }\end{array}$ & 1855 & concentrated & 50.78 \\
\hline \multicolumn{10}{|c|}{ 8. Total Expense } \\
\hline CR1 & 40.61 & $\begin{array}{l}\text { moderate } \\
\text { market share }\end{array}$ & 37.88 & $\begin{array}{l}\text { moderate } \\
\text { market share }\end{array}$ & 31.53 & $\begin{array}{l}\text { moderate } \\
\text { market share }\end{array}$ & 29.52 & $\begin{array}{l}\text { low market } \\
\text { share }\end{array}$ & $(6.38)$ \\
\hline CR4 & 71.82 & tight oligopoly & 69.23 & tight oligopoly & 62.21 & tight oligopoly & 61.84 & tight oligopoly & $(0.599)$ \\
\hline HHI & 2120 & concentrated & 1931 & concentrated & 1494 & $\begin{array}{l}\text { moderate } \\
\text { concentrated }\end{array}$ & 1421 & $\begin{array}{l}\text { moderate } \\
\text { concentrated }\end{array}$ & $(4.89)$ \\
\hline \multicolumn{10}{|c|}{ 9. Profit from Operation } \\
\hline CR1 & 68.57 & $\begin{array}{l}\text { high market } \\
\text { share }\end{array}$ & 62.76 & $\begin{array}{l}\text { high market } \\
\text { share }\end{array}$ & 44.93 & $\begin{array}{l}\text { moderate } \\
\text { market share }\end{array}$ & 38.98 & $\begin{array}{l}\text { moderate market } \\
\text { share }\end{array}$ & $(13.25)$ \\
\hline CR4 & 87.15 & tight oligopoly & 86.26 & tight oligopoly & 75.51 & tight oligopoly & 74.43 & tight oligopoly & $(1.43)$ \\
\hline HHI & 4864 & $\begin{array}{l}\text { highly } \\
\text { concentrated }\end{array}$ & 4196 & $\begin{array}{l}\text { highly } \\
\text { concentrated }\end{array}$ & 2487 & concentrated & 2099 & concentrated & $(15.58)$ \\
\hline
\end{tabular}

Source: Calculated by author, using data from Office of Insurance Commission 

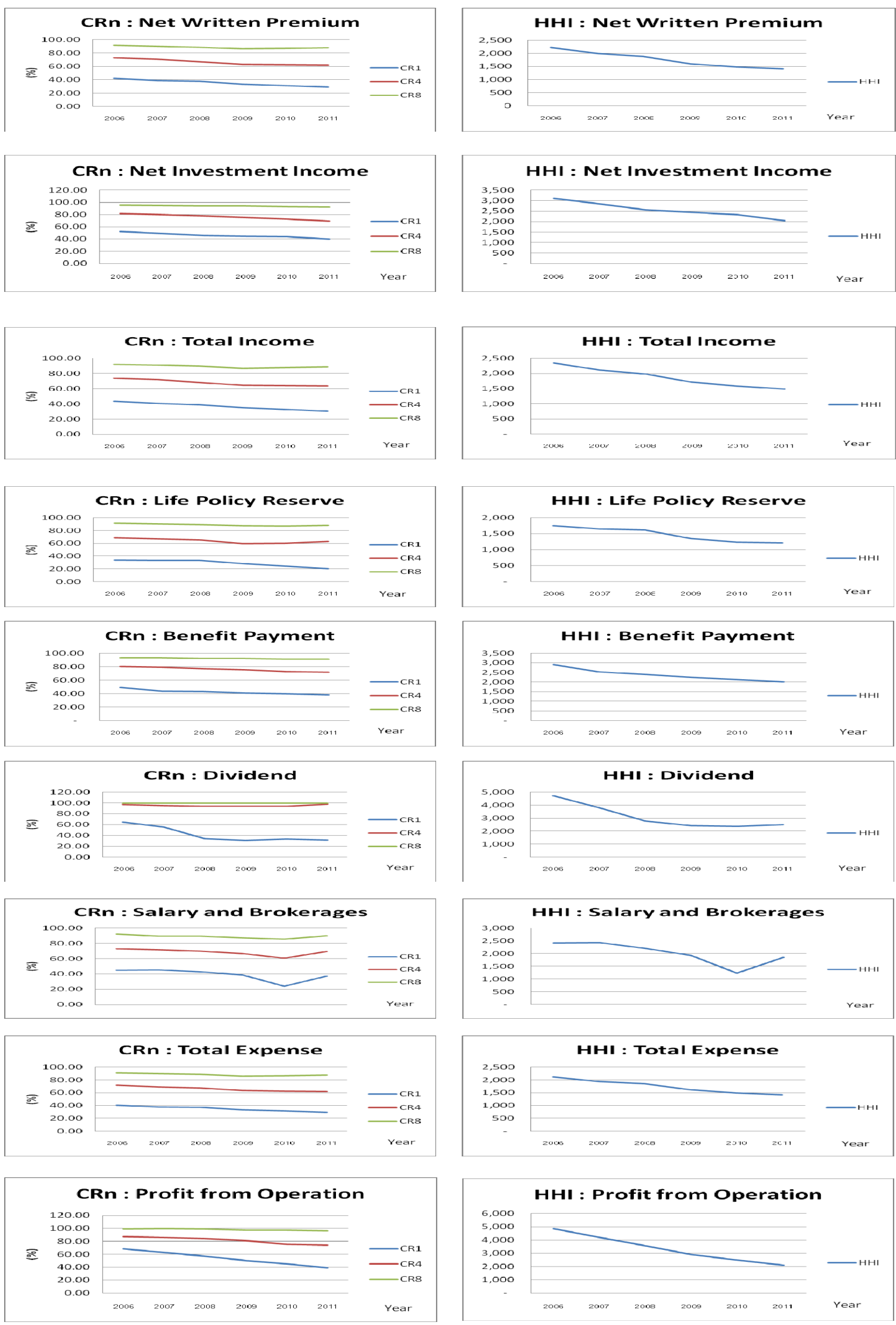

Figure 3. Market concentration on life insurance income statement

Source: Calculated by author, using data from Office of Insurance Commission 
As shown in Table 5 and Figure 3, the life insurance income statement is tight oligopoly and concentrated. The $\mathrm{CR}_{4}$ and $\mathrm{HHI}$ of net written premium are $62.21 \%$ and 1407 , respectively, in 2011 . The $\mathrm{CR}_{4}$ and $\mathrm{HHI}$ of life policy reserve are $62.67 \%$ and 1208 , respectively, in 2011 . The $\mathrm{CR}_{4}$ and $\mathrm{HHI}$ of profit from operation are $74.43 \%$ and 2099, respectively, in 2011.

\subsection{Non-Life Insurance Industry}

This part presents market concentration on non-life insurance premium, non-life insurance balance sheet and non-life insurance income statement.

3.2.1 Market Concentrations on Non-Life Insurance Premium

Table 6. Market concentration on non-life insurance premium

\begin{tabular}{|c|c|c|c|c|c|c|c|c|c|c|c|}
\hline \multicolumn{2}{|c|}{ Year } & \multicolumn{3}{|c|}{1997} & \multicolumn{3}{|l|}{1998} & 2010 & \multicolumn{2}{|r|}{2011} & $\begin{array}{l}\text { \% increas } \\
\text { (decrease) }\end{array}$ \\
\hline \multicolumn{12}{|c|}{ 1. Total Premium } \\
\hline CR1 & 13.13 & $\begin{array}{l}\text { low } \\
\text { share }\end{array}$ & market & 12.27 & $\begin{array}{l}\text { low } \\
\text { share }\end{array}$ & market & 16.15 & market & 15.51 & low market share & (3.94) \\
\hline CR4 & 34.08 & monop & olistic & 31.50 & monop & listic & 36.71 & monopolistic & 37.03 & monopolistic & 0.88 \\
\hline HHI & 467 & uncon & entrated & 419 & uncon & ntrated & 533 & unconcentrated & 531 & unconcentrated & $(0.38)$ \\
\hline
\end{tabular}

\section{Fire Premium}

\begin{tabular}{|c|c|c|c|c|c|c|c|c|c|}
\hline CR1 & 12.18 & $\begin{array}{l}\text { low } \\
\text { share }\end{array}$ & 12.09 & $\begin{array}{l}\text { low } \\
\text { share }\end{array}$ & 16.15 & $\begin{array}{l}\text { low } \\
\text { share }\end{array}$ & 12.74 & low market share & (21.13) \\
\hline CR4 & 32.15 & monopolistic & 32.18 & monopolistic & 36.71 & monopolistic & 42.56 & loose oligopoly & 15.94 \\
\hline HHI & 435 & unconcentrated & 429 & unconcentrated & 533 & unconcentrated & 640 & unconcentrated & 20.08 \\
\hline
\end{tabular}

\section{Marine and Transportation Premium}

\begin{tabular}{|c|c|c|c|c|c|c|c|c|c|}
\hline CR1 & 11.42 & $\begin{array}{l}\text { low } \\
\text { share }\end{array}$ & 10.93 & $\begin{array}{l}\text { low } \\
\text { share }\end{array}$ & 14.57 & $\begin{array}{l}\text { low } \\
\text { share }\end{array}$ & 12.93 & low market share & (11.28) \\
\hline CR4 & 33.63 & monopolistic & 33.90 & monopolistic & 42.18 & loose oligopoly & 39.81 & monopolistic & $(5.61)$ \\
\hline HHI & 475 & unconcentrated & 471 & unconcentrated & 648 & unconcentrated & 585 & unconcentrated & $(9.72)$ \\
\hline
\end{tabular}

\section{Automobile Premium}

$\begin{array}{lllllllllll}\text { CR1 } & 20.35 & \begin{array}{l}\text { low market } \\ \text { share }\end{array} & 20.13 & \begin{array}{l}\text { low } \\ \text { share }\end{array} & \text { market } & 24.92 & \begin{array}{l}\text { low } \\ \text { share }\end{array} & \text { market } & 23.98 & \text { low market share } \\ \text { CR4 } & 50.02 & \text { loose oligopoly } & 43.58 & \text { loose oligopoly } & 42.61 & \text { loose oligopoly } & 41.74 & \text { loose oligopoly } \\ \text { HHI } & 821 & \text { unconcentrated } & 734 & \text { unconcentrated } & 858 & \text { unconcentrated } & 828 & \text { unconcentrated }\end{array}$

\section{Personal Accident Premium}

\begin{tabular}{|c|c|c|c|c|c|c|c|c|}
\hline CR1 & \multirow{3}{*}{ n.a. } & 45.71 & $\begin{array}{l}\text { moderate } \\
\text { market share }\end{array}$ & 19.58 & $\begin{array}{l}\text { low market } \\
\text { share }\end{array}$ & 26.32 & low market share & 34.43 \\
\hline CR4 & & 62.68 & tight oligopoly & 50.76 & loose oligopoly & 53.09 & loose oligopoly & 4.60 \\
\hline HHI & & 2244 & concentrated & 944 & unconcentrated & 1109 & moderate concentrated & 17.48 \\
\hline \multicolumn{9}{|c|}{ 6. Health Premium } \\
\hline CR1 & \multirow{3}{*}{ n.a. } & 47.12 & $\begin{array}{l}\text { moderate } \\
\text { market share }\end{array}$ & 31.30 & $\begin{array}{l}\text { moderate } \\
\text { market share }\end{array}$ & 30.72 & moderate market share & $(1.87)$ \\
\hline CR4 & & 77.18 & tight oligopoly & 60.54 & tight oligopoly & 61.23 & tight oligopoly & 1.14 \\
\hline HHI & & 2640 & $\begin{array}{l}\text { highly } \\
\text { concentrated }\end{array}$ & 1396 & $\begin{array}{l}\text { moderate } \\
\text { concentrated }\end{array}$ & 1369 & moderate concentrated & $(1.93)$ \\
\hline
\end{tabular}

Source: Calculated by author, using data from Office of Insurance Commission 

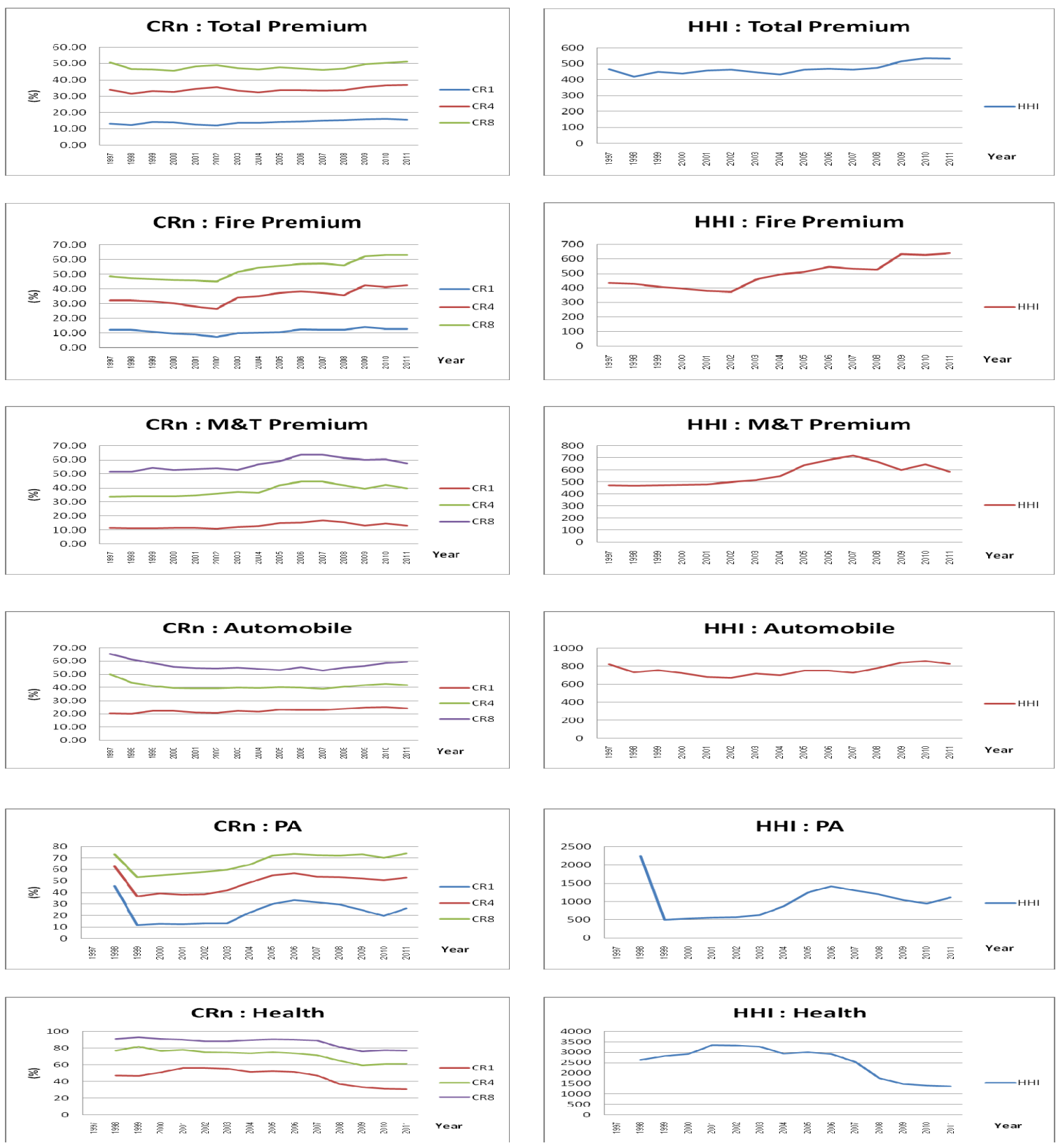

Figure 4. Market concentrations on direct non-life insurance premium

Source: Calculated by author, using data from Office of Insurance Commission

As shown in Table 6 and Figure 4, the non-life insurance industry in term of total premium indicates monopolistic competition and unconcentrated market. The $\mathrm{CR}_{4}$ and $\mathrm{HHI}$ of total premium are $37.03 \%$ and 531 , respectively, in 2011. Non-life insurance industry in Thailand has four sub-categorized products, including fire, marine and transportation, automobile, personal accident and health in miscellaneous insurance product. The highest concentration is health product. The $\mathrm{CR}_{4}$ and $\mathrm{HHI}$ of health premium is $61.23 \%$ and 1369 , respectively, in 2011. However, the HHI trend of the health market concentration slows down. 
3.2.2 Market Concentrations on Non-Life Insurance Balance Sheet

Table 7. Market concentration on non-life insurance balance sheet

\begin{tabular}{|c|c|c|c|c|c|c|c|c|c|c|}
\hline Year & & 2006 & & 2007 & & 2010 & & 2011 & \multicolumn{2}{|c|}{$\begin{array}{l}\text { \% increas } \\
\text { (decrease) }\end{array}$} \\
\hline \multicolumn{11}{|c|}{ 1. Investment Assets } \\
\hline CR1 & 16.74 & $\begin{array}{l}\text { low market } \\
\text { share }\end{array}$ & 16.47 & $\begin{array}{l}\text { low market } \\
\text { share }\end{array}$ & 14.89 & low market share & 16.03 & low $\mathrm{m}$ & t share & 7.64 \\
\hline CR4 & 42.21 & loose oligopoly & 41.71 & loose oligopoly & 43.08 & loose oligopoly & 44.53 & loose & poly & 3.37 \\
\hline HHI & 631 & unconcentrated & 625 & unconcentrated & 641 & unconcentrated & 697 & uncon & rated & 8.63 \\
\hline
\end{tabular}

\section{Building \& Operating Assets}

\begin{tabular}{|c|c|c|c|c|c|c|c|c|}
\hline CR1 & 13.92 & $\begin{array}{l}\text { low } \\
\text { share }\end{array}$ & 13.36 & $\begin{array}{l}\text { low } \\
\text { share }\end{array}$ & 18.09 & low market share & 13.47 & low market share \\
\hline CR4 & 40.34 & loose oligopoly & 39.00 & monopolistic & 44.20 & loose oligopoly & 38.17 & monopolistic \\
\hline HHI & 614 & unconcentrated & 591 & unconcentrated & 721 & unconcentrated & 614 & unconcentrated \\
\hline
\end{tabular}

\section{Others Assets}

\begin{tabular}{|c|c|c|c|c|c|c|c|c|c|}
\hline CR1 & 10.96 & market & 11.20 & $\begin{array}{l}\text { low } \\
\text { share }\end{array}$ & 17.67 & low market share & 24.48 & low market share & 38.53 \\
\hline CR4 & 35.63 & monopolistic & 31.62 & monopolistic & 46.92 & loose oligopoly & 53.38 & loose oligopoly & 13. \\
\hline HHI & 460 & unconcentrated & 406 & unconcentrated & 768 & unconcentrated & 991 & unconcentrated & \\
\hline
\end{tabular}

\section{Total Asset}

\begin{tabular}{|c|c|c|c|c|c|c|c|c|c|}
\hline CR1 & 15.23 & $\begin{array}{l}\text { low } \\
\text { share }\end{array}$ & 14.65 & $\begin{array}{l}\text { low } \\
\text { share }\end{array}$ & 15.54 & low market share & 19.56 & low market share & 25.87 \\
\hline CR4 & 38.60 & monopolistic & 37.70 & monopolistic & 43.12 & loose oligopoly & 43.85 & loose oligopoly & 1.69 \\
\hline HHI & 545 & unconcentrated & 533 & unconcentrated & 638 & unconcentrated & 729 & unconcentrated & 14.16 \\
\hline
\end{tabular}

\section{Policy Liabilities}

\begin{tabular}{|c|c|c|c|c|c|c|c|c|c|}
\hline CR1 & 19.69 & $\begin{array}{l}\text { low } \\
\text { share }\end{array}$ & 20.15 & $\begin{array}{l}\text { low } \\
\text { share }\end{array}$ & 19.36 & low market share & 22.39 & low market share & 15.67 \\
\hline CR4 & 39.94 & monopolistic & 39.55 & monopolistic & 42.10 & loose oligopoly & 50.30 & loose oligopoly & 19.46 \\
\hline HHI & 649 & unconcentrated & 649 & unconcentrated & 683 & unconcentrated & 880 & unconcentrated & 28.84 \\
\hline
\end{tabular}

\section{Other Liabilities}

$\begin{array}{llllllllll}\text { CR1 } & 16.75 & \begin{array}{l}\text { low } \\ \text { share }\end{array} & 16.23 & \begin{array}{l}\text { low } \\ \text { share }\end{array} & \text { market } & 33.66 & \begin{array}{l}\text { moderate market } \\ \text { share }\end{array} & 23.80 & \text { low market share } \\ \text { CR4 } & 36.97 & \text { monopolistic } & 35.21 & \text { monopolistic } & 52.45 & \text { loose oligopoly } & 41.83 & \text { loose oligopoly } \\ \text { HHI } & 542 & \text { unconcentrated } & 519 & \text { unconcentrated } & 1,357 & \begin{array}{l}\text { moderate } \\ \text { concentrated }\end{array} & 797 & \text { unconcentrated }\end{array}$

\section{Capital Funds}

\begin{tabular}{|c|c|c|c|c|c|c|c|c|c|}
\hline CR1 & 26.14 & market & 26.07 & $\begin{array}{l}\text { low market } \\
\text { share }\end{array}$ & 24.41 & low market share & 23.44 & low market share & $(10.31)$ \\
\hline CR4 & 48.37 & loose oligopoly & 49.15 & loose oligopoly & 47.43 & loose oligopoly & 54.14 & loose oligopoly & 11.92 \\
\hline HHI & 967 & unconcentrated & 971 & unconcentrated & 892 & unconcentrated & 1060 & $\begin{array}{l}\text { moderate } \\
\text { concentrated }\end{array}$ & 9.59 \\
\hline
\end{tabular}

Source: Calculated by author, using data from Office of Insurance Commission 

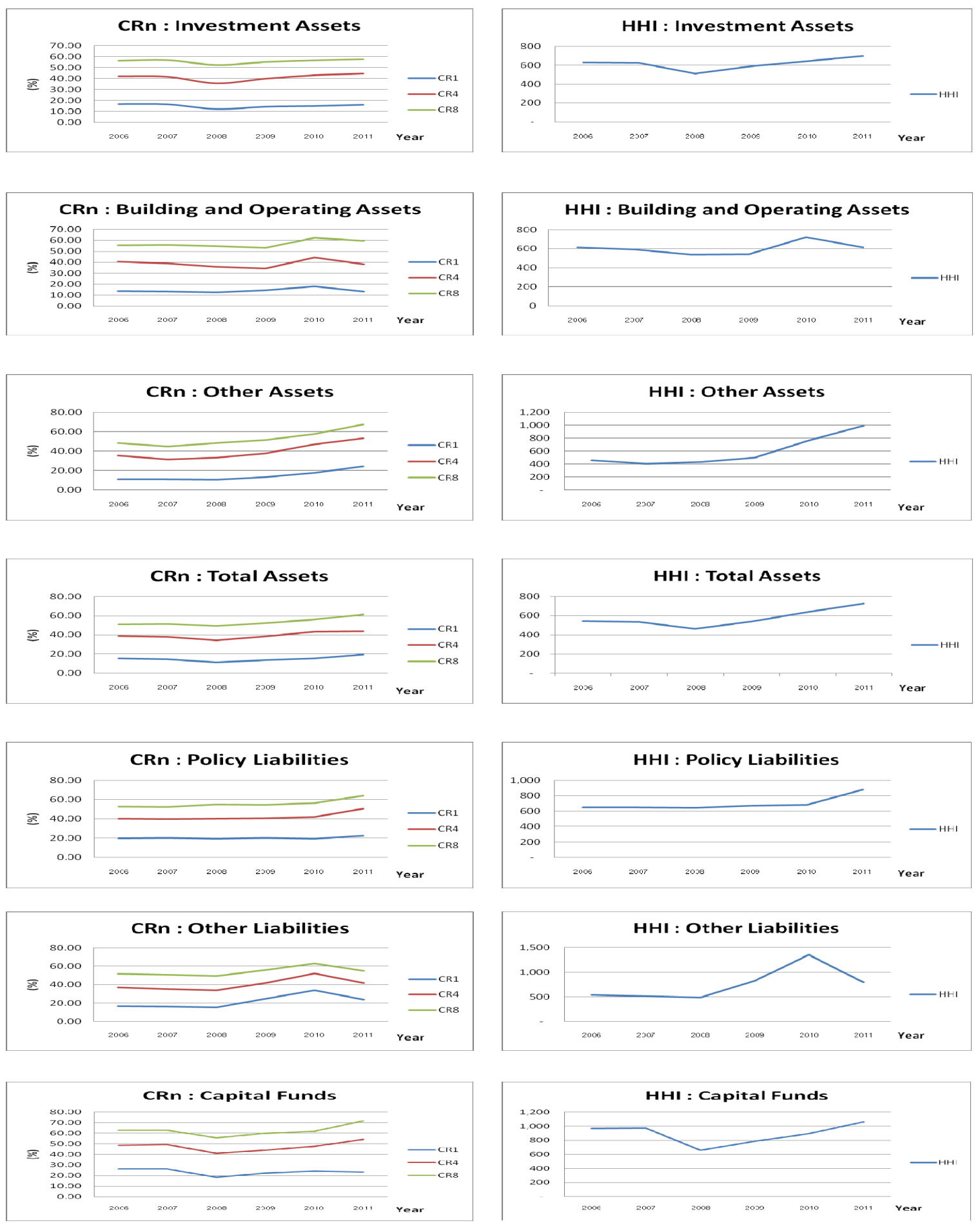

Figure 5. Market concentrations on non-life insurance balance sheet

Source: Calculated by author, using data from Office of Insurance Commission

As shown in Table 7 and Figure 5, the non-life insurance balance sheet is loose oligopoly and unconcentrated with increasing trend. The $\mathrm{CR}_{4}$ and $\mathrm{HHI}$ of investment asset are $44.53 \%$ and 697 , respectively, in 2011 . The $\mathrm{CR}_{4}$ and $\mathrm{HHI}$ of total asset are $43.85 \%$ and 729 , respectively, in 2011 . The $\mathrm{CR}_{4}$ and $\mathrm{HHI}$ of policy liabilities are $50.30 \%$ and 880 , respectively, in 2011 . The $\mathrm{CR}_{4}$ and $\mathrm{HHI}$ of capital funds are $23.44 \%$ and 1060 , respectively, in 2011. 
3.2.3 Market Concentrations on Non-Life Insurance Income Statement

Table 8. Market concentration on non-life insurance income statement

\begin{tabular}{|c|c|c|c|c|c|c|c|c|c|}
\hline Year & & 2006 & & 2007 & & 2010 & & 2011 & $\begin{array}{l}\% \text { inc } \\
\text { (decrea }\end{array}$ \\
\hline \multicolumn{10}{|c|}{ 1. Earn Premiums } \\
\hline CR1 & $\begin{array}{l}18.9 \\
8\end{array}$ & $\begin{array}{l}\text { low market } \\
\text { share }\end{array}$ & 20.01 & market & $\begin{array}{l}20.4 \\
8\end{array}$ & market & $\begin{array}{l}20.7 \\
0\end{array}$ & $\begin{array}{l}\text { low market } \\
\text { share }\end{array}$ & 1.08 \\
\hline CR4 & $\begin{array}{l}35.5 \\
6\end{array}$ & monopolistic & 36.58 & monopolistic & $\begin{array}{l}38.0 \\
9\end{array}$ & monopolistic & $\begin{array}{l}38.2 \\
3\end{array}$ & monopolistic & 0.38 \\
\hline HHI & 575 & $\begin{array}{l}\text { unconcentrate } \\
\text { d }\end{array}$ & 615 & $\begin{array}{l}\text { unconcentrate } \\
\text { d }\end{array}$ & 655 & $\begin{array}{l}\text { unconcentrate } \\
\text { d }\end{array}$ & 672 & $\begin{array}{l}\text { unconcentrate } \\
\text { d }\end{array}$ & 2.64 \\
\hline
\end{tabular}

\section{Net Investment and other income}

\begin{tabular}{|c|c|c|c|c|c|c|c|c|}
\hline CR1 & $\begin{array}{l}13.2 \\
2\end{array}$ & market & 12.49 & $\begin{array}{l}\text { low } \\
\text { share }\end{array}$ & $\begin{array}{l}18.7 \\
8\end{array}$ & $\begin{array}{l}\text { low } \\
\text { share }\end{array}$ & $\begin{array}{l}15.7 \\
4\end{array}$ & $\begin{array}{l}\text { low } \\
\text { share }\end{array}$ \\
\hline CR4 & $\begin{array}{l}36.0 \\
3\end{array}$ & monopolistic & 34.59 & monopolistic & $\begin{array}{l}49.7 \\
1\end{array}$ & $\begin{array}{l}\text { loose } \\
\text { oligopoly }\end{array}$ & $\begin{array}{l}44.2 \\
0\end{array}$ & $\begin{array}{l}\text { loose } \\
\text { oligopoly }\end{array}$ \\
\hline HHI & 517 & $\begin{array}{l}\text { unconcentrate } \\
\text { d }\end{array}$ & 486 & $\begin{array}{l}\text { unconcentrate } \\
\text { d }\end{array}$ & 793 & $\begin{array}{l}\text { unconcentrate } \\
\text { d }\end{array}$ & 674 & $\begin{array}{l}\text { unconcentrate } \\
\text { d }\end{array}$ \\
\hline
\end{tabular}

\section{Total Income}

\begin{tabular}{|c|c|c|c|c|c|c|c|c|}
\hline CR1 & $\begin{array}{l}18.4 \\
6\end{array}$ & market & 19.35 & $\begin{array}{l}\text { low } \\
\text { share }\end{array}$ & $\begin{array}{l}19.8 \\
6\end{array}$ & $\begin{array}{l}\text { low } \\
\text { share }\end{array}$ & $\begin{array}{l}19.9 \\
0\end{array}$ & $\begin{array}{l}\text { low } \\
\text { share }\end{array}$ \\
\hline CR4 & $\begin{array}{l}35.2 \\
2\end{array}$ & monopolistic & 36.03 & monopolistic & $\begin{array}{l}37.5 \\
1\end{array}$ & monopolistic & $\begin{array}{l}37.6 \\
6\end{array}$ & monopolistic \\
\hline HHI & 558 & $\begin{array}{l}\text { unconcentrate } \\
\text { d }\end{array}$ & 593 & $\begin{array}{l}\text { unconcentrate } \\
\text { d }\end{array}$ & 635 & $\begin{array}{l}\text { unconcentrate } \\
\text { d }\end{array}$ & 647 & $\begin{array}{l}\text { unconcentrate } \\
\text { d }\end{array}$ \\
\hline
\end{tabular}

\section{Losses Incurred after Deduction}

\begin{tabular}{|c|c|c|c|c|c|c|c|c|c|}
\hline CR1 & $\begin{array}{l}21.6 \\
5\end{array}$ & $\begin{array}{l}\text { low market } \\
\text { share }\end{array}$ & 22.26 & $\begin{array}{l}\text { low } \\
\text { share }\end{array}$ & $\begin{array}{l}21.3 \\
6\end{array}$ & $\begin{array}{l}\text { low } \\
\text { share }\end{array}$ & $\begin{array}{l}64.1 \\
4\end{array}$ & $\begin{array}{l}\text { high } \\
\text { share }\end{array}$ & 200.36 \\
\hline CR4 & $\begin{array}{l}39.6 \\
7\end{array}$ & monopolistic & 40.70 & $\begin{array}{l}\text { loose } \\
\text { oligopoly }\end{array}$ & $\begin{array}{l}39.0 \\
9\end{array}$ & monopolistic & $\begin{array}{l}76.2 \\
3\end{array}$ & tight oligopoly & 95.03 \\
\hline $\mathrm{HHI}$ & 687 & $\begin{array}{l}\text { unconcentrate } \\
\text { d }\end{array}$ & 718 & $\begin{array}{l}\text { unconcentrate } \\
\text { d }\end{array}$ & 695 & $\begin{array}{l}\text { unconcentrate } \\
\text { d }\end{array}$ & 4193 & $\begin{array}{l}\text { highly } \\
\text { concentrated }\end{array}$ & 503.76 \\
\hline
\end{tabular}

\section{Commission and Brokerages}

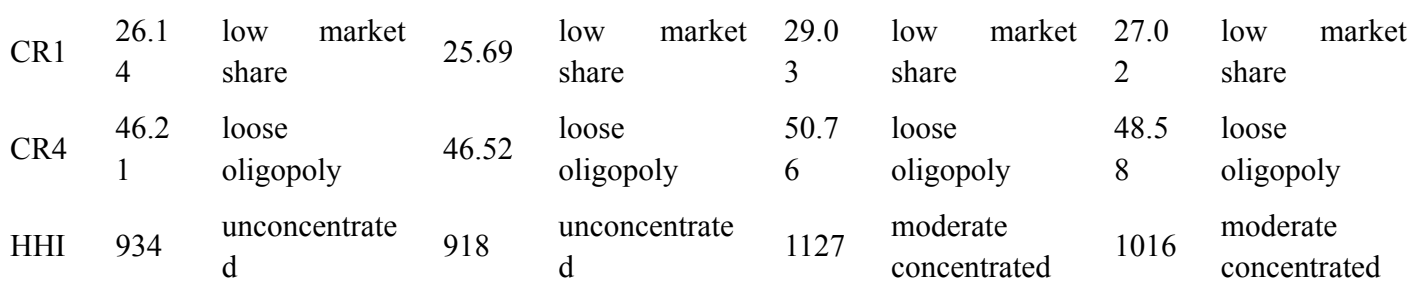

\section{Underwriting Expenses}

\begin{tabular}{|c|c|c|c|c|c|c|c|c|}
\hline CR1 & $\begin{array}{l}14.2 \\
8\end{array}$ & $\begin{array}{l}\text { low } \\
\text { share }\end{array}$ & $\begin{array}{l}15.6 \\
3\end{array}$ & $\begin{array}{l}\text { low } \\
\text { share }\end{array}$ & 19.41 & $\begin{array}{l}\text { low } \\
\text { share }\end{array}$ & $\begin{array}{l}18.3 \\
6\end{array}$ & $\begin{array}{l}\text { low } \\
\text { share }\end{array}$ \\
\hline CR4 & $\begin{array}{l}50.9 \\
6\end{array}$ & $\begin{array}{l}\text { loose } \\
\text { oligopoly }\end{array}$ & $\begin{array}{l}50.6 \\
1\end{array}$ & loose oligopoly & 53.66 & $\begin{array}{l}\text { loose } \\
\text { oligopoly }\end{array}$ & $\begin{array}{l}40.4 \\
3\end{array}$ & $\begin{array}{l}\text { loose } \\
\text { oligopoly }\end{array}$ \\
\hline HHI & 788 & $\begin{array}{l}\text { unconcentrate } \\
\text { d }\end{array}$ & 801 & unconcentrated & 946 & $\begin{array}{l}\text { unconcentrate } \\
\text { d }\end{array}$ & 695 & $\begin{array}{l}\text { unconcentrate } \\
\text { d }\end{array}$ \\
\hline
\end{tabular}




\begin{tabular}{|c|c|c|c|c|c|}
\hline Year & 2006 & 2007 & 2010 & 2011 & $\begin{array}{l}\% \text { increase } \\
\text { (decrease) }\end{array}$ \\
\hline
\end{tabular}

\section{Operating Expenses}

\begin{tabular}{|c|c|c|c|c|c|c|c|c|}
\hline CR1 & $\begin{array}{l}13.2 \\
9\end{array}$ & $\begin{array}{l}\text { low } \\
\text { share }\end{array}$ & $\begin{array}{l}13.5 \\
5\end{array}$ & $\begin{array}{l}\text { low market } \\
\text { share }\end{array}$ & 13.81 & $\begin{array}{l}\text { low } \\
\text { share }\end{array}$ & $\begin{array}{l}14.5 \\
0\end{array}$ & market \\
\hline CR4 & $\begin{array}{l}26.0 \\
7\end{array}$ & monopolistic & $\begin{array}{l}25.5 \\
8\end{array}$ & monopolistic & 28.45 & monopolistic & $\begin{array}{l}29.8 \\
7\end{array}$ & monopolistic \\
\hline HHI & 367 & $\begin{array}{l}\text { unconcentrate } \\
\text { d }\end{array}$ & 370 & $\begin{array}{l}\text { unconcentrate } \\
\text { d }\end{array}$ & 404 & $\begin{array}{l}\text { unconcentrate } \\
\text { d }\end{array}$ & 429 & unconcentrated \\
\hline
\end{tabular}

\section{Total Expense}

\begin{tabular}{|c|c|c|c|c|c|c|c|c|c|}
\hline CR1 & $\begin{array}{l}19.9 \\
2\end{array}$ & $\begin{array}{l}\text { low } \\
\text { share }\end{array}$ & $\begin{array}{l}20.3 \\
1\end{array}$ & $\begin{array}{l}\text { low market } \\
\text { share }\end{array}$ & 20.65 & $\begin{array}{l}\text { low } \\
\text { share }\end{array}$ & $\begin{array}{l}52.8 \\
6\end{array}$ & $\begin{array}{l}\text { high } \\
\text { share }\end{array}$ & 155.96 \\
\hline CR4 & $\begin{array}{l}36.6 \\
1\end{array}$ & monopolistic & $\begin{array}{l}37.1 \\
8\end{array}$ & monopolistic & 37.09 & monopolistic & $\begin{array}{l}68.0 \\
1\end{array}$ & tight oligopoly & 83.36 \\
\hline HHI & 606 & $\begin{array}{l}\text { unconcentrate } \\
\text { d }\end{array}$ & 623 & $\begin{array}{l}\text { unconcentrate } \\
\text { d }\end{array}$ & 645 & $\begin{array}{l}\text { unconcentrate } \\
\text { d }\end{array}$ & 2927 & $\begin{array}{l}\text { highly } \\
\text { concentrated }\end{array}$ & 353.62 \\
\hline
\end{tabular}

\section{Profit from Operation}

\begin{tabular}{|c|c|c|c|c|c|c|c|c|c|}
\hline CR1 & $\begin{array}{l}12.9 \\
9\end{array}$ & $\begin{array}{l}\text { low } \\
\text { share }\end{array}$ & $\begin{array}{l}11.2 \\
2\end{array}$ & $\begin{array}{l}\text { low market } \\
\text { share }\end{array}$ & 16.55 & $\begin{array}{l}\text { low } \\
\text { share }\end{array}$ & $\begin{array}{l}22.9 \\
2\end{array}$ & $\begin{array}{l}\text { low } \\
\text { share }\end{array}$ & 38.44 \\
\hline CR4 & $\begin{array}{l}40.3 \\
5\end{array}$ & $\begin{array}{l}\text { loose } \\
\text { oligopoly }\end{array}$ & $\begin{array}{l}39.2 \\
1\end{array}$ & monopolistic & 41.92 & $\begin{array}{l}\text { loose } \\
\text { oligopoly }\end{array}$ & $\begin{array}{l}63.1 \\
2\end{array}$ & tight oligopoly & 50.58 \\
\hline IHI & 618 & $\begin{array}{l}\text { unconcentrate } \\
\text { d }\end{array}$ & 613 & $\begin{array}{l}\text { unconcentrate } \\
\text { d }\end{array}$ & 678 & $\begin{array}{l}\text { unconcentrate } \\
\text { d }\end{array}$ & 1227 & $\begin{array}{l}\text { moderate } \\
\text { concentrated }\end{array}$ & 80.96 \\
\hline
\end{tabular}

Source: Calculated by author, using data from Office of Insurance Commission
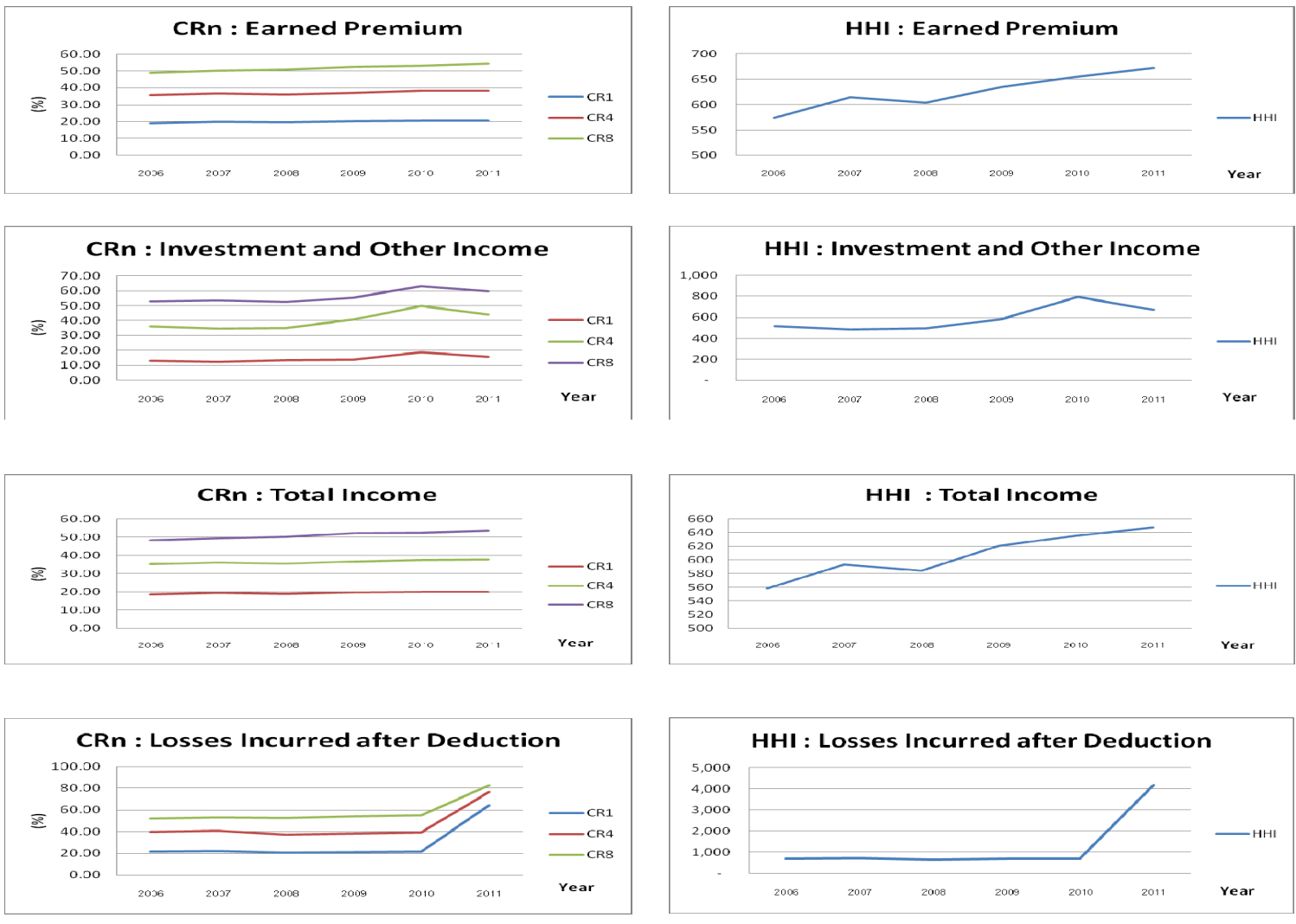

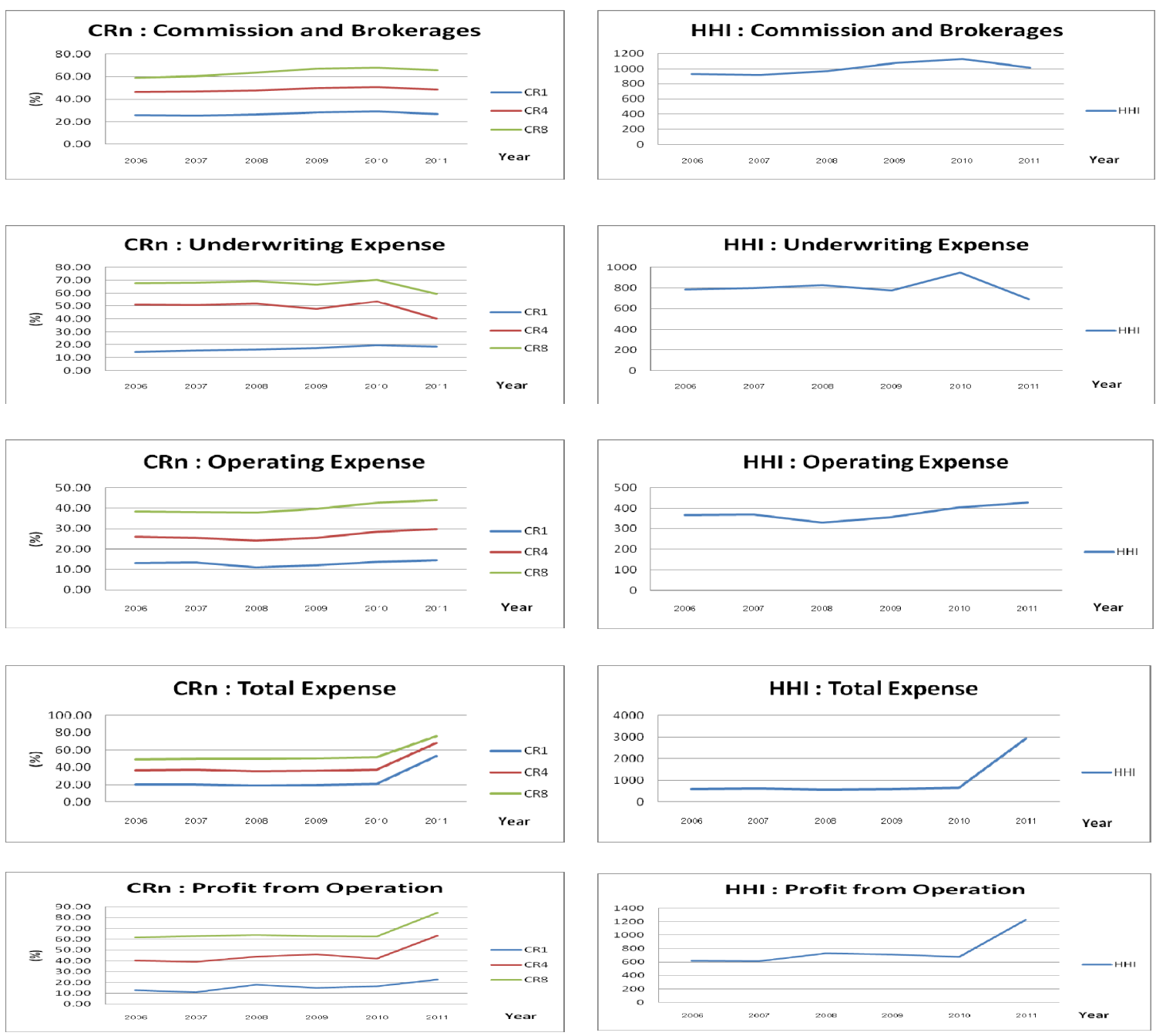

Figure 6. Market concentrations on non-life insurance income statement

Source: Calculated by author, using data from Office of Insurance Commission

As shown in Table 8 and Figure 6, the non-life insurance income statement in term of earn premium is monopolistic competition and unconcentrated whereas profit from operation is tight oligopoly and concentrated with increasing trend. The $\mathrm{CR}_{4}$ and $\mathrm{HHI}$ of earn premiums are $38.23 \%$ and 672 , respectively, in 2011 . The $\mathrm{CR}_{4}$ and HHI of total asset are $68.41 \%$ and 1962 , respectively, in 2011 . The $\mathrm{CR}_{4}$ and $\mathrm{HHI}$ of policy liabilities are $66.15 \%$ and 1718 , respectively, in 2011 . The $\mathrm{CR}_{4}$ and $\mathrm{HHI}$ of profit from operation are $63.12 \%$ and 1227 , respectively, in 2011.

3.3 Comparative on Personal Accident (PA) and Health between Life and Non-Life Insurance

This part presents market concentration on the same sub-categorized product in life and non-life industry as personal accident and health insurance. 
Table 9. Comparative on PA and Health between life and non-life insurance

\begin{tabular}{|c|c|c|c|c|c|c|c|c|c|}
\hline \multicolumn{2}{|l|}{ Year } & 2004 & & 2005 & & 2010 & & 2011 & $\begin{array}{l}\% \\
\text { increase } \\
\text { (decrease) }\end{array}$ \\
\hline \multicolumn{10}{|c|}{ 1. Personal Accident Premium } \\
\hline \multicolumn{10}{|c|}{ 1.1 Life Insurance } \\
\hline CR1 & 83.52 & $\begin{array}{l}\text { high market } \\
\text { share }\end{array}$ & 81.13 & $\begin{array}{l}\text { high market } \\
\text { share }\end{array}$ & 74.55 & $\begin{array}{l}\text { high market } \\
\text { share }\end{array}$ & 74.45 & $\begin{array}{l}\text { high market } \\
\text { share }\end{array}$ & $(0.14)$ \\
\hline CR4 & 92.96 & near monopoly & 90.98 & $\begin{array}{l}\text { near } \\
\text { monopoly }\end{array}$ & 90.13 & near monopoly & 91.76 & $\begin{array}{l}\text { near } \\
\text { monopoly }\end{array}$ & 1.80 \\
\hline HHI & 7016 & $\begin{array}{l}\text { highly } \\
\text { concentrated }\end{array}$ & 6629 & $\begin{array}{l}\text { highly } \\
\text { concentrated }\end{array}$ & 5664 & $\begin{array}{l}\text { highly } \\
\text { concentrated }\end{array}$ & 5662 & $\begin{array}{l}\text { highly } \\
\text { concentrated }\end{array}$ & $(0.04)$ \\
\hline \multicolumn{10}{|c|}{ 1.2 Non-Life Insurance } \\
\hline CR1 & 22.37 & $\begin{array}{l}\text { low market } \\
\text { share }\end{array}$ & 30.02 & $\begin{array}{l}\text { moderate } \\
\text { market share }\end{array}$ & 19.58 & $\begin{array}{l}\text { low market } \\
\text { share }\end{array}$ & 26.32 & $\begin{array}{l}\text { low market } \\
\text { share }\end{array}$ & 34.43 \\
\hline CR4 & 48.42 & $\begin{array}{l}\text { loose } \\
\text { oligopoly }\end{array}$ & 54.86 & $\begin{array}{l}\text { loose } \\
\text { oligopoly }\end{array}$ & 50.76 & $\begin{array}{l}\text { loose } \\
\text { oligopoly }\end{array}$ & 53.09 & $\begin{array}{l}\text { loose } \\
\text { oligopoly }\end{array}$ & 4.60 \\
\hline HHI & 878 & unconcentrated & 1241 & $\begin{array}{l}\text { moderate } \\
\text { concentrated }\end{array}$ & 944 & unconcentrated & 1109 & $\begin{array}{l}\text { moderate } \\
\text { concentrated }\end{array}$ & 17.48 \\
\hline \multicolumn{10}{|c|}{ 2. Health Premium } \\
\hline \multicolumn{10}{|c|}{ 2.1 Life Insurance } \\
\hline CR1 & 55.79 & $\begin{array}{l}\text { high market } \\
\text { share }\end{array}$ & 48.72 & $\begin{array}{l}\text { moderate } \\
\text { market share }\end{array}$ & 49.34 & $\begin{array}{l}\text { moderate } \\
\text { market share }\end{array}$ & 48.23 & $\begin{array}{l}\text { moderate } \\
\text { market share }\end{array}$ & $(2.24)$ \\
\hline CR4 & 88.35 & tight oligopoly & 86.64 & $\begin{array}{l}\text { tight } \\
\text { oligopoly }\end{array}$ & 83.79 & tight oligopoly & 83.13 & $\begin{array}{l}\text { tight } \\
\text { oligopoly }\end{array}$ & $(0.79)$ \\
\hline HHI & 3543 & $\begin{array}{l}\text { highly } \\
\text { concentrated }\end{array}$ & 2914 & $\begin{array}{l}\text { highly } \\
\text { concentrated }\end{array}$ & 2906 & $\begin{array}{l}\text { highly } \\
\text { concentrated }\end{array}$ & 2804 & $\begin{array}{l}\text { highly } \\
\text { concentrated }\end{array}$ & $(3.51)$ \\
\hline \multicolumn{10}{|c|}{ 2.2 Non-Life Insurance } \\
\hline CR1 & 51.52 & $\begin{array}{l}\text { high market } \\
\text { share }\end{array}$ & 52.24 & $\begin{array}{l}\text { high market } \\
\text { share }\end{array}$ & 31.30 & $\begin{array}{l}\text { moderate } \\
\text { market share }\end{array}$ & 30.72 & $\begin{array}{l}\text { moderate } \\
\text { market share }\end{array}$ & $(1.87)$ \\
\hline CR4 & 73.58 & tight oligopoly & 74.96 & $\begin{array}{l}\text { tight } \\
\text { oligopoly }\end{array}$ & 60.54 & tight oligopoly & 61.23 & $\begin{array}{l}\text { tight } \\
\text { oligopoly }\end{array}$ & 1.14 \\
\hline HHI & 2941 & $\begin{array}{l}\text { highly } \\
\text { concentrated }\end{array}$ & 3008 & $\begin{array}{l}\text { highly } \\
\text { concentrated }\end{array}$ & 1396 & $\begin{array}{l}\text { moderate } \\
\text { concentrated }\end{array}$ & 1369 & $\begin{array}{l}\text { moderate } \\
\text { concentrated }\end{array}$ & $(1.93)$ \\
\hline
\end{tabular}

Source: Calculated by author, using data from Office of Insurance Commission
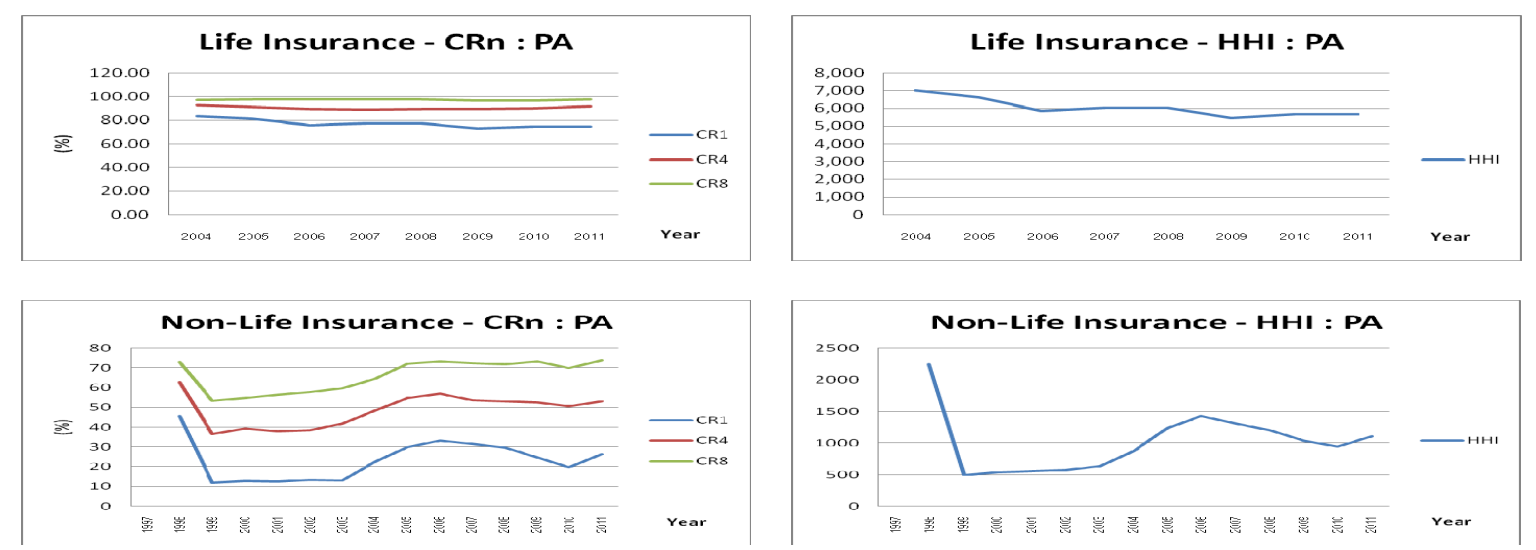

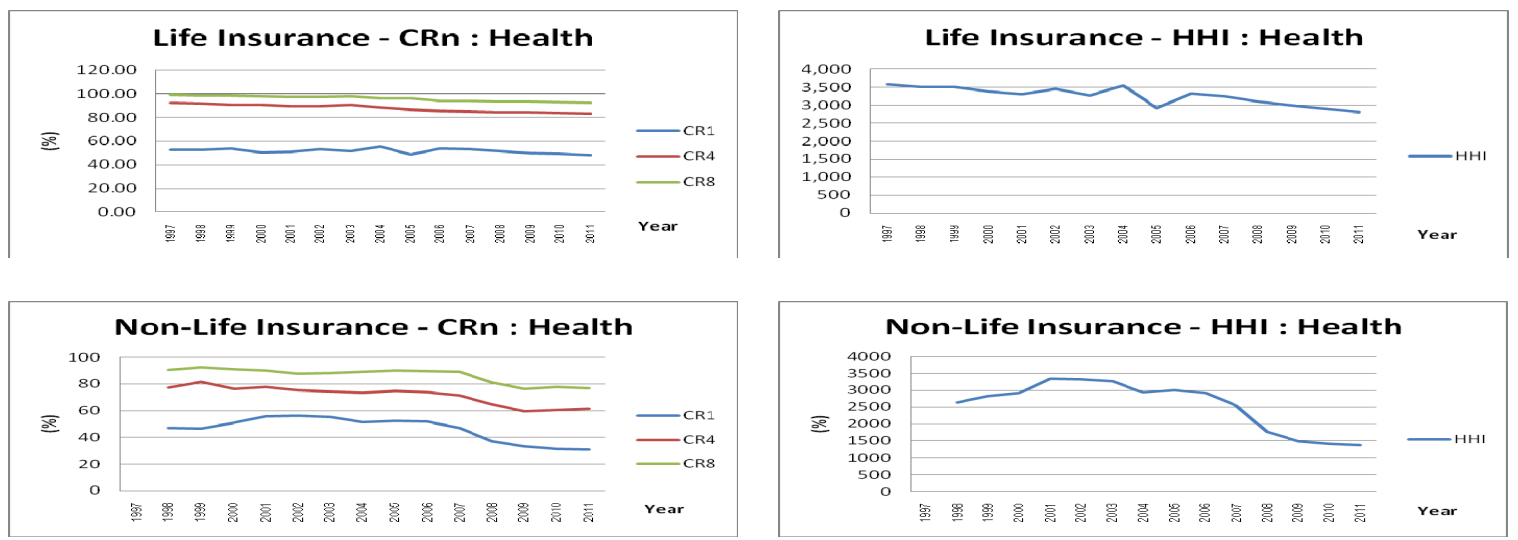

Figure 7. Market concentration on PA and health insurance

Source: Calculated by author, using data from Office of Insurance Commission

As shown in Table 9 and Figure 7, the same sub-categorized product, PA and health, in life and non-life industry have difference concentrate degree. PA in life insurance is near monopoly and highly concentrated market while $\mathrm{PA}$ in non-life insurance is loose oligopoly and moderate concentrated market. The $\mathrm{CR}_{4}$ and $\mathrm{HHI}$ of PA in life insurance are $91.76 \%$ and 5662, respectively, in 2011. The $\mathrm{CR}_{4}$ and $\mathrm{HHI}$ of PA in non-life insurance are 53.09\% and 1109, respectively, in 2011. Health in life and non-life insurance are the same, tight oligopoly that concentrate on large four firm. The $\mathrm{CR}_{4}$ and $\mathrm{HHI}$ of health in life insurance are $83.13 \%$ and 2804 , respectively, in 2011. The $\mathrm{CR}_{4}$ and $\mathrm{HHI}$ of health in non-life insurance are $61.23 \%$ and 1369 , respectively, in 2011. Health in life is more concentrated than health in non-life insurance. However, the trend of the health market concentration slows down that more competition in life and non-life insurance industry.

\section{Conclusions}

Using concentration ratio and Herfindahl-Hirschman index, it can be concluded the life insurance industry in Thailand is tight oligopoly and moderately concentrated. There are 24 life insurance companies in Thailand. The $\mathrm{CR}_{4}$ and $\mathrm{HHI}$ of total premium are $62.08 \%$ and 1402 , respectively, in 2011. Life insurance industry in Thailand has four sub-categorized products including ordinary, industry, group, personal accident and health insurance. The highest competition is group product and the highest concentration is industry product. An analysis of life insurance balance sheet and income statement shows similar results which are concentrated. However, all of concentration trends are slowing down, non-price competition is likely to increase. Customers always buy insurance from insurance company which has reputation and good after-sales service.

Using concentration ratio and Herfindahl-Hirschman index, it can be concluded the non-life insurance industry is monopolistic competition and unconcentrated. There are 65 non-life insurance companies in Thailand. The $\mathrm{CR}_{4}$ and $\mathrm{HHI}$ of total premium are $37.03 \%$ and 531, respectively, in 2011. Non-life insurance industry in Thailand has four sub-categorized product under fire, marine and transportation, automobile, personal accident and health in miscellaneous insurance. The fire, marine and transportation, automobile and personal accident insurance markets are unconcentrated where as health insurance market is moderate concentrated. An analysis of non-life insurance balance sheet and income statement show similar results which are unconcentrated market. However, all of trends are upward because some companies exit from the non-life insurance market. There are 74 companies, 73 companies, 73 companies, 71 companies, 69 companies and 68 companies in period from 2006 to 2011.

The same sub-categorized product in life and non-life industry, personal accident and health products have difference concentration degrees. Personal accident in life insurance is near monopoly while personal accident in non-life insurance is loose oligopoly. Health in life and non-life insurance have similar results, which are tight oligopoly. Consumers will purchase personal accident and health insurance from insurance company which has intensive hospital networks and good after-sales service to ensure getting the best treatment. Every company attempts to improve their service so all concentration trends slow down, leading to the reduction in market concentration in life and non-life insurance industry.

By concentration measurement, Concentration Ratio and the Herfindahl-Hirschman Index, this paper concludes that life insurance industry is more concentrated than non-life insurance industry. In both segments of the 
insurance market, as well as in the overall insurance sector, there is a downward trend in market concentration, which indicates the success of the competition promoting process for benefiting consumers the most. However, alongside competition, it is necessary for government to conduct proper regulation in order to avoid adverse market situations.

\section{References}

Austin, D. A., \& Hungerford, T. L. (2009). The Market Structure of the Health Insurance Industry. Congressional Research Service Report. The United States.

Bajtelsmit, V. L., \& Bouzouita, R. (1998). Market Structure and Performance in Private Passenger Automobile Insurance. The Journal of Risk and Insurance, 65(3), 503-514. Colorado. The United States. http://dx.doi.org/10.2307/253662

Cummins, J. D. (2008). The Bermuda Insurance Market: An Economic Analysis. The World's Risk Capital Report. Bermuda.

Dumm, R. E., \& Macpherson, D. A. (2006). A Preliminary Comparative Financial Analysis of Title Insurance Company. Report of Office of Insurance Regulation. Florida.

Ehrlich, I., \& Becker, G. S. (1972). Market Insurance, Self-Insurance, and Self-Protection. The Journal of Political Economy, 89(4), 623-648. Chicago. The United States. http://dx.doi.org/10.1086/259916

Feen et al. (2006). Market Structure and the efficiency of European Insurance companies: Stochastic Frontier analysis. KPMG Business Measurement Research. The United of Kingdom.

Glambotskaya, A. (2009). The Belarusian Insurance Market Characteristics in the context of Economic liberalization: Analysis and Policy Recommendations. Policy Paper, 1, 1-12. Minsk, Belarus.

Greguric et al. (2011). Liberalization and Market Concentration in the Insurance Industry: Case of Croatia. Journal of Economics and Business, 9(2), 61-75. Croatia.

Ishii, H. (2009). The Insurance Market in 2009: Changing Channels and Markets. The Toa Reinsurance Company, Limited. Japan.

Jedlicka, L., \& Jumah, A. (2006). The Austrian Insurance Industry: A structure, conduct and performance analysis. Economic Series, 189, 1-39. Vienna, Austrian.

Johnson, D. F., \& Stone, G. (1998). Introduction to Economics. United States of America: Life Office Management Association, Inc. C.

Kozarevic, S., Regan, L., \& Gibbons, R. J. (2008). The European Union Integration Process and Insurance Market Development: The Case of the Former Yoguslav Countries. International Insurance Foundation. The United States.

Lipczynski, J., Wilson, J., \& Goddard, J. (2005). Industrial Organization, Competition, Strategy, Policy. Harlow: Prentice Hall.

Milo, M. S. (2003). State of Competition in the insurance industry: Selected Asian Countries. The Research Information Staff, Philippine Institute for Development Studies. Philippines.

Nissan, E., \& Caveny, R. (2001). A Comparison of Large Firm Dominance in Property and Liability Insurance with Major Industries. Journal of Insurance Issues, 24(1 \& 2), 58-73. Mississippi. The United States.

Office of Insurance Commission. (2012). Statistic Report. Retrieved from http://www.oic.or.th

Office of the National Economic and Social Development Board. (2010). Statistic Report. Retrieved from http://www.nesdb.go.th

\section{Copyrights}

Copyright for this article is retained by the author(s), with first publication rights granted to the journal.

This is an open-access article distributed under the terms and conditions of the Creative Commons Attribution license (http://creativecommons.org/licenses/by/3.0/). 